\title{
1 The Structural Basis of Rubisco Phase Separation in the Pyrenoid
}

3 Shan $\mathrm{He}^{1}$, Hui-Ting $\mathrm{Chou}^{2}$, Doreen Matthies $^{2}$, Tobias Wunder ${ }^{3}$, Moritz T. Meyer ${ }^{1}$, Nicky

4 Atkinson $^{4}$, Antonio Martinez-Sanchez ${ }^{5,6}$, Philip D. Jeffrey ${ }^{1}$, Sarah A. Port ${ }^{1}$, Weronika Patena ${ }^{1}$,

5 Guanhua He ${ }^{1}$, Vivian K. Chen ${ }^{7}$, Frederick M. Hughson ${ }^{1}$, Alistair J. McCormick ${ }^{4}$, Oliver Mueller-

6 Cajar $^{3}$, Benjamin D. Engel ${ }^{5,8}$, Zhiheng $\mathrm{Yu}^{2}$, Martin C. Jonikas ${ }^{1, *}$

$8 \quad{ }^{1}$ Department of Molecular Biology, Princeton University, Princeton, NJ 08544, USA. ${ }^{2}$ Janelia

9 Research Campus, Howard Hughes Medical Institute, 19700 Helix Drive, Ashburn, VA 20147,

10 USA. ${ }^{3}$ School of Biological Sciences, Nanyang Technological University, 60 Nanyang Drive,

11 Singapore 637551, Singapore. ${ }^{4}$ SynthSys \& Institute of Molecular Plant Sciences, School of

12 Biological Sciences, University of Edinburgh, Edinburgh, EH9 3BF, UK. ${ }^{5}$ Department of

13 Molecular Structural Biology, Max Planck Institute of Biochemistry, 82152 Martinsried,

14 Germany. ${ }^{6}$ Institute of Neuropathology, University of Göttingen Medical Center, 37075

15 Göttingen, Germany. ${ }^{7}$ Department of Biology, Stanford University, Stanford, CA 94305, USA.

$16{ }^{8}$ Helmholtz Pioneer Campus, Helmholtz Zentrum München, 85764 Neuherberg, Germany.

$17{ }^{*}$ Correspondence and Lead Contact: mjonikas@princeton.edu. 


\section{Abstract}

20 Approximately one-third of global $\mathrm{CO}_{2}$ fixation occurs in a phase separated algal organelle called

21 the pyrenoid. Existing data suggest that the pyrenoid forms by the phase-separation of the $\mathrm{CO}_{2}-$

22 fixing enzyme Rubisco with a linker protein; however, the molecular interactions underlying this

23 phase-separation remain unknown. Here we present the structural basis of the interactions between

24 Rubisco and its intrinsically disordered linker protein EPYC1 (Essential Pyrenoid Component 1)

25 in the model alga Chlamydomonas reinhardtii. We find that EPYC1 consists of five evenly-spaced

26 Rubisco-binding regions that share sequence similarity. Single-particle cryo-electron microscopy

27 of one of these regions in complex with Rubisco indicates that each Rubisco holoenzyme has eight

28 binding sites for EPYC1, one on each Rubisco small subunit. Interface mutations disrupt binding,

29 phase separation, and pyrenoid formation. Cryo-electron tomography supports a model where

30 EPYC1 and Rubisco form a co-dependent multivalent network of specific low-affinity bonds,

31 giving the matrix liquid-like properties. Our results advance the structural and functional

32 understanding of the phase separation underlying the pyrenoid, an organelle that plays a

33 fundamental role in the global carbon cycle. 


\section{Main Text}

The $\mathrm{CO}_{2}$-fixing enzyme Rubisco drives the global carbon cycle, mediating the assimilation

36 of approximately 100 gigatons of carbon per year ${ }^{1}$. The gradual decrease of atmospheric $\mathrm{CO}_{2}$ over

37 billions of years ${ }^{2}$ has made Rubisco's job increasingly difficult, to the point where $\mathrm{CO}_{2}$

38 assimilation limits the growth rate of many photosynthetic organisms ${ }^{3}$. This selective pressure is

39 thought to have led to the evolution of $\mathrm{CO}_{2}$ concentrating mechanisms, which feed concentrated

$40 \mathrm{CO}_{2}$ to Rubisco to enhance growth ${ }^{4}$. Of these mechanisms, the most poorly understood relies on

41 the pyrenoid, a phase separated organelle ${ }^{5}$ found in the chloroplast of nearly all eukaryotic algae

42 and some land plants (Fig. 1a, b) ${ }^{6,7}$. The pyrenoid enhances the activity of Rubisco by clustering

43 it around modified thylakoid membranes that supply Rubisco with concentrated $\mathrm{CO}_{2}{ }^{8,9}$.

44 For decades, the mechanism for packaging the Rubisco holoenzyme into the pyrenoid

45 remained unknown. Recent work showed that in the leading model alga Chlamydomonas

46 reinhardtii (Chlamydomonas hereafter), the clustering of Rubisco into the pyrenoid matrix

47 requires the Rubisco-binding protein EPYC1 ${ }^{10}$. EPYC1 and Rubisco are the most abundant

48 components of the pyrenoid and bind to each other. Moreover, combining purified EPYC1 and

49 Rubisco together produces phase-separated condensates ${ }^{11}$ that mix internally at a rate similar to

50 that observed for the matrix in vivo ${ }^{5}$, suggesting that these two proteins are sufficient to form the

51 structure of the pyrenoid matrix. The sequence repeats within EPYC1 and eight-fold symmetry of

52 the Rubisco holoenzyme led us to hypothesize that EPYC1 and Rubisco each have multiple

53 binding sites for the other, allowing the two proteins to form a co-dependent condensate (Fig. 1c) ${ }^{10}$.

$54 \quad$ Here, we determined the structural basis that underlies the EPYC1-Rubisco condensate.

55 Using biophysical approaches, we found that EPYC1 has five evenly spaced Rubisco-binding

56 regions that share sequence homology and can bind to Rubisco as short peptides. We obtained a

57 cryo-electron microscopy structure, which shows that each of EPYC1's Rubisco-binding regions

58 forms an $\alpha$-helix that binds one of Rubisco's eight small subunits via salt bridges and hydrophobic

59 interactions. Mapping of these binding sites onto Rubisco holoenzymes within the native pyrenoid

60 matrix indicates that the linker sequences between Rubisco-binding regions on EPYC1 are

61 sufficiently long to connect together adjacent Rubisco holoenzymes. These discoveries advance

62 the understanding of the pyrenoid, and provide a high resolution structural view of a phase-

63 separated organelle. 


\section{Results}

\section{EPYC1 has five nearly-identical Rubisco-binding regions}

67 We could not directly determine the structure of full-length EPYC1 bound to Rubisco because mixing the two proteins together produces phase separated condensates ${ }^{11}$. We thus aimed to first identify Rubisco-binding regions on EPYC1, and subsequently to use a structural approach to determine how these regions bind to Rubisco.

The intrinsically disordered nature of purified $E P Y C 1^{11}$ led us to hypothesize that the Rubisco-binding regions of EPYC1 were short and could bind to Rubisco as peptides without a need for tertiary folds. Therefore, to identify EPYC1 regions that bind to Rubisco, we synthesized

74 a peptide array consisting of 18 amino acid peptides tiling across the full length EPYC1 sequence 75 (Fig. 1d), and probed this array with native Rubisco purified from Chlamydomonas cells (Fig. 1e, $76 \mathrm{f})$.

Our tiling array revealed five evenly-spaced Rubisco-binding regions on EPYC1, each consisting of a predicted $\alpha$-helix and an upstream region (Fig. $1 \mathrm{~g}, \mathrm{~h}$ ). We confirmed the binding regions using surface plasmon resonance (SPR; Extended Data Fig. 1b, c ). Sequence alignment guided by the five binding regions revealed that mature EPYC1 consists entirely of five sequence repeats (Fig. 1i), in contrast to the previously defined four repeats and two termini ${ }^{10}$ (Extended Data Fig. 1a). Our alignment indicates that the previously defined EPYC1 N- and C- termini, which at the time were not considered part of the repeats, actually share sequence homology with the central repeats.

The presence of a Rubisco-binding region on each of the previously defined EPYC1 repeats (Extended Data Fig. 1a) explains our yeast two-hybrid observations ${ }^{12}$ that a single EPYC1 repeat can interact with Rubisco, that knocking out the $\alpha$-helix in an EPYC1 repeat disrupts this interaction, and that decreasing the number of EPYC1 repeats leads to a proportional decrease in

89 EPYC1 interaction with Rubisco. It also explains our observations that decreasing the number of 90 EPYC1 repeats leads to a proportional decrease in the tendency of EPYC1 and Rubisco to phase 91 separate together ${ }^{11}$.

EPYC1 binds to Rubisco small subunits

94 The sequence homology of the five Rubisco-binding regions suggests that each region binds to

95 Rubisco in a similar manner. To identify the binding site of EPYC1 on Rubisco, we performed 
single-particle cryo-electron microscopy on a complex of Rubisco and a peptide corresponding to the first Rubisco-binding region of EPYC1 (Fig. 2a). We selected this region of EPYC1 because in preliminary experiments it had the highest affinity to Rubisco, which was still low by protein interaction standards ( $\mathrm{K}_{\mathrm{D}} \sim 3 \mathrm{mM}$; Extended Data Fig. 1d, e). This low affinity meant that millimolar concentrations of peptide were required to approach full occupancy of peptide bound to Rubisco, leading to challenges with peptide insolubility and high background signal in the electron micrographs. Despite these challenges, we successfully obtained a $2.62 \AA$ structure of the complex ( 2.9 Å EPYC1 peptide local resolution; Fig. 2, Extended Data Fig. 2 and 3; Extended Data Table 1). For reference purposes, we also obtained a $2.68 \AA$ cryo-electron density map of Rubisco in the absence of EPYC1 peptide (Extended Data Fig. 2), which was nearly identical to the previously published X-ray crystallography structure ${ }^{13}$, with minor differences likely due to the absence of the substrate analog 2-CABP in the active site of Rubisco in our sample ${ }^{14}$ (Extended Data Fig. 4).

The Rubisco holoenzyme consists of a core of eight catalytic large subunits in complex with eight small subunits, four of which cap each end of the holoenzyme (Fig. 2b-e). In our structure, an EPYC1 peptide was clearly visible bound to each Rubisco small subunit, suggesting

\section{Binding is mediated by salt bridges and a hydrophobic interface}

115 The EPYC1 peptide forms an extended chain that sits on top of the Rubisco small subunit's two $116 \alpha$-helices (Fig. 3a, b). The structure explains our previous observations that mutations in the 117 Rubisco small subunit $\alpha$-helices disrupted yeast two-hybrid interactions between EPYC1 and the 118 Rubisco small subunit ${ }^{12}$ and prevented Rubisco's assembly into a pyrenoid in vivo ${ }^{15}$. The C119 terminal region of the EPYC1 peptide (NWRQELESLRN) is well-resolved and forms an $\alpha$-helix 120 that runs parallel to helix B of the Rubisco small subunit (Fig. 3a, b). The peptide's N-terminus 121 extends the trajectory of the helix and follows the surface of the Rubisco small subunit (Fig. 2b-e,

122 3a-b and Extended Data Fig. 5a). The side chains of the peptide's N-terminus could not be well 123 resolved, suggesting that this region is more conformationally flexible.

124 Our atomic model based on the density map suggests that binding is mediated by salt 125 bridges and a hydrophobic interface. Three residue pairs likely form salt bridges (Fig. 3c, d and 
127 subunit $\alpha$-helix A, and EPYC1 residue E66 interacts with R91 of Rubisco small subunit $\alpha$-helix B.

128 Furthermore, a hydrophobic interface is formed by W63, L67 and L70 of EPYC1 and M87, L90

129 and V94 of Rubisco small subunit helix B (Fig. 3e-g).

\section{Interface residues are required for binding and phase separation in vitro}

132 To determine the importance of individual EPYC1 residues for binding, we investigated the impact 133 on Rubisco binding of every possible single amino acid substitution for EPYC1's first Rubisco134 binding region by using a peptide array (Fig. 4a) and SPR (Extended Data Fig. 5b). Consistent 135 with our structural model, the peptide array indicated that EPYC1 salt bridge-forming residues 136 R64, R71 and E66 and the hydrophobic interface residues W63, L67 and L70 were all required for 137 normal EPYC1 binding to Rubisco. The strong agreement of our mutational analysis suggests that 138 our structural model correctly represents EPYC1's Rubisco-binding interface.

139 To determine the importance of EPYC1's Rubisco-binding regions for pyrenoid matrix 140 formation, we assayed the impact of mutations in these regions on formation of phase separated 141 droplets by EPYC1 and Rubisco in vitro. The phase boundary was shifted by mutating R64 in the 142 first Rubisco-binding region and the corresponding $\mathrm{K}$ or $\mathrm{R}$ in the other four Rubisco-binding 143 regions of EPYC1 (Fig. 4b and Extended Data Fig. 5c-e), suggesting that the Rubisco-binding 144 regions mediate condensate formation.

\section{The binding interface is required for pyrenoid matrix formation in vivo}

147 We validated the importance of Rubisco residues for binding to EPYC1 by yeast two-hybrid assays

148 (Fig. 5a and Extended Data Fig. 6). Rubisco small subunit D23A mutation, which eliminates the 149 charge of that residue, had a severe impact on Rubisco small subunit interaction with EPYC1, as 150 expected from the contribution of that residue to a salt bridge with R71 of EPYC1. Likewise, E24A 151 and R91A each showed a moderate defect, consistent with the contributions of those residues to 152 salt bridges with R64 and E66 of EPYC1, respectively. Additionally, M87D and V94D, which 153 convert hydrophobic residues to bulky charged residues, each had a severe impact on interaction, 154 as expected from the participation of those residues in the hydrophobic interface. Combinations of 155 these mutations abolished the interactions completely (Extended Data Fig. 6).

156 To evaluate the importance of the binding interface in vivo, we generated Chlamydomonas 157 strains with point mutations in the binding interface. Rubisco small subunit mutations D23A/E24A 
or M87D/V94D caused a growth defect under conditions requiring a functional pyrenoid (Fig. 5b,

159 Extended Data Fig. 7a-b). Furthermore, the mutants lacked a visible pyrenoid matrix (Fig. 5c, d

160 and Extended Data Fig. 7c), indicating that those Rubisco small subunit residues are required for

161 matrix formation in vivo. The Rubisco mutants retained pyrenoid tubules ${ }^{16}$, as previously observed

162 in other matrix-deficient mutants ${ }^{10,17-19}$.

163 Together, our data demonstrate that EPYC1's Rubisco-binding regions bind to the Rubisco

164 small subunit $\alpha$-helices via salt-bridge interactions and a hydrophobic interface, enabling the

165 condensation of Rubisco into the phase separated matrix.

The spacing between EPYC1's Rubisco-binding regions allows linking of adjacent Rubisco holoenzymes in the native pyrenoid matrix

169 The presence of multiple Rubisco-binding regions along the EPYC1 sequence supports a model 170 where consecutive Rubisco-binding regions on the same EPYC1 polypeptide can bind to different 171 Rubisco holoenzymes and thus hold them together to form the pyrenoid matrix. If this model is 172 correct, we would expect that the $\sim 40$ amino acid "linker" regions between consecutive Rubisco173 binding regions on EPYC1 (Fig. 1g, i) would be sufficient to span the distance between EPYC1174 binding sites on neighboring Rubisco holoenzymes in the pyrenoid matrix. To test this aspect of 175 the model, we combined our atomic structure of the EPYC1-Rubisco interaction with the precise 176 positions and orientations of Rubisco holoenzymes within the pyrenoid matrix of native cells that 177 we had previously obtained by in-situ cryo-electron tomography ${ }^{5}$ (Fig. 6a, b). We mapped the 178 positions of EPYC1 binding sites onto Rubisco holoenzymes in the matrix and measured the 179 distances between nearest neighbor EPYC1 binding sites on adjacent holoenzymes (Fig. 6c). The 180 observed distances ranged from $\sim 2 \mathrm{~nm}$ to $\sim 7 \mathrm{~nm}$, with a median distance of $\sim 4 \mathrm{~nm}$ (Fig. $6 \mathrm{~d}$ ).

A "linker" region of 40 amino acids is unlikely to be stretched to its maximum possible

182 length of $14 \mathrm{~nm}$ in vivo due to the high entropic cost of this configuration. To determine whether 183 a linker region can span the observed distances between nearest neighbor binding sites on adjacent 184 Rubisco holoenzymes, we used a simple physics model to calculate the energy required to stretch 185 a 40 amino acid chain to any given distance (Fig. 6d; see Methods). The model indicates that 186 stretching the chain to $\sim 7 \mathrm{~nm}$ requires an energy of $3 k_{\mathrm{B}} T$ (where $k_{\mathrm{B}}$ is the Boltzmann constant and $187 T$ is the temperature), which could reasonably be borrowed from thermal fluctuations. Thus, our 188 data suggest that the linker region between consecutive Rubisco-binding sites on the EPYC1 
189 polypeptide can span the distance between adjacent Rubisco holoenzymes to hold the pyrenoid 190 matrix together in vivo. It also appears likely that, in addition to bridging neighboring Rubisco

191 holoenzymes, consecutive Rubisco-binding regions on a given EPYC1 may bind multiple sites on

192 one Rubisco holoenzyme, as the distance between the nearest binding sites on the same 193 holoenzyme is $<9 \mathrm{~nm}$.

\section{Discussion}

\section{Our data explain the structural basis of Rubisco condensation into a pyrenoid matrix}

197 In this study, we have determined the structural basis for pyrenoid matrix formation for the first 198 time in any species. We found that in the model alga Chlamydomonas, the intrinsically disordered 199 protein EPYC1 has five regions of similar sequence that can bind to Rubisco as short peptides.

200 These EPYC1 regions form an $\alpha$-helix that binds to the Rubisco small subunit $\alpha$-helices via salt 201 bridges and hydrophobic interactions. EPYC1's Rubisco-binding regions are spaced by linker 202 sequences that are sufficiently long to span the distance between binding sites on adjacent Rubisco 203 holoenzymes within the pyrenoid, allowing EPYC1 to serve as a molecular glue that clusters 204 Rubisco together to form the pyrenoid matrix (Fig. 6e).

205 The multivalency of EPYC1 and the high $\mathrm{K}_{\mathrm{D}}(\sim 3 \mathrm{mM}$; Extended Data Fig. 1e) of individual 206 Rubisco-binding regions are consistent with the emerging principle that cellular phase separation 207 is mediated by weak multivalent interactions ${ }^{20}$. The high dissociation rate constant $(>1 / \mathrm{s}$; Extended 208 Data Fig. 1d) of individual Rubisco-binding regions explains how the pyrenoid matrix can mix 209 internally on the time scale of seconds ${ }^{5}$ despite the multivalency of EPYC1. The even spacing of 210 the five Rubisco-binding regions across EPYC1 is noteworthy and may be an indication of 211 selective pressure for an optimal distance between binding regions, and thus of an optimal spacing 212 between Rubisco holoenzymes in the matrix.

213 Knowledge of the EPYC1-Rubisco binding mechanism now opens doors to the molecular 214 characterization of the regulation of this interaction, which may govern the dissolution and 215 condensation of the matrix during cell division ${ }^{5}$ and in response to environmental factors ${ }^{21}$. For 216 example, phosphorylation of $\mathrm{EPYC1}^{22}$ may provide a mechanism to rapidly change the binding 217 affinity of EPYC1 to Rubisco. Inactivation of one of EPYC1's five Rubisco-binding regions would 218 yield four binding regions, which would allow two EPYC1 molecules to form a mutually satisfied 219 complex with each Rubisco, a configuration that is predicted to favor dissolution of the matrix ${ }^{5}$. 


\section{The Rubisco-EPYC1 structure explains how other key pyrenoid proteins bind to Rubisco}

222 In a parallel study (Meyer et al., please see unpublished manuscript provided as reference 223 material), we recently discovered that a common sequence motif is present on many pyrenoid224 localized proteins. The motif binds Rubisco, enabling recruitment of motif-containing proteins to 225 the pyrenoid and mediating adhesion between the matrix, pyrenoid tubules, and starch sheath. This 226 motif, [D/N]W[R/K]XX[L/I/V/A], is serendipitously present in EPYC1's Rubisco-binding 227 regions, and the motif residues mediate key binding interactions with Rubisco. In our structure, 228 the $\mathrm{R} / \mathrm{K}$ of the motif is represented by R64 of EPYC1, which forms a salt bridge with E24 of the 229 Rubisco small subunit. The XX of the motif almost always includes a D or E; in our structure this 230 feature is represented by E66 of EPYC1, which forms a salt bridge with R91 of the Rubisco small 231 subunit. Finally, the $\mathrm{W}$ and [L/I/V/A] of the motif are represented by W63 and L67 of EPYC1, 232 which contribute to the hydrophobic interactions with M87, L90 and V94 of the Rubisco small 233 subunit. The key roles of the motif residues in the interface presented here strongly suggest that 234 the structure we have obtained for one motif from EPYC1 also explains where and how all other 235 variants of the motif, including those found on the key pyrenoid proteins SAGA1, SAGA2, 236 RBMP1, RBMP2 and CSP41A, bind to Rubisco. Our observation that the Rubisco small subunit $237 \mathrm{D} 23 \mathrm{~A} / \mathrm{E} 24 \mathrm{~A}$ and M87D/V94D mutants exhibit a more severe disruption of the pyrenoid than the 238 epycl mutant ${ }^{10}$ supports the idea that this region of Rubisco interacts not only with EPYC1, but 239 also with other proteins required for pyrenoid biogenesis, making this binding interaction a central 240 hub of pyrenoid biogenesis.

242 There are structural similarities and differences between the pyrenoid matrix and bacterial 243 carboxysomes

244 Although $\alpha$ - and $\beta$-carboxysomes are morphologically, functionally and evolutionarily distinct 245 from the pyrenoid, their Rubisco is also thought to be clustered by linker proteins. Like EPYC1, 246 the $\alpha$-carboxysome linker protein $\mathrm{CsoS} 2^{23}$ is intrinsically disordered and is proposed to bind 247 Rubisco as an unfolded peptide ${ }^{24}$. In contrast, the $\beta$-carboxysome linker protein CcmM has been 248 proposed to bind Rubisco using folded globular domains ${ }^{25,26}$. The use of an unfolded peptide as in 249 the case of EPYC1 and CsoS2 may provide the benefit of requiring fewer amino acids for 250 achieving the desired binding function. A notable difference is the location of the binding site on 
251 Rubisco: whereas both carboxysomal linker proteins bind to the interface between two Rubisco

252 large subunits ${ }^{24,26}$, EPYC1 binds to the Rubisco small subunit. It remains to be seen whether this

253 difference in binding site has functional consequences, such as impacts on the three-dimensional

254 packing of Rubisco.

Our findings advance the ability to engineer a pyrenoid into crops

257 There is currently significant interest in engineering Rubisco condensates into monocotyledonous 258 crops such as wheat and rice to enhance yields ${ }^{27-30}$. Binding of EPYC1 to the Rubisco small subunit

259 presents a promising route for engineering a Rubisco condensate, as the Rubisco small subunit is

260 encoded in the nuclear genome, making it more easily amenable to genetic modification in those 261 crops than the chloroplast-encoded Rubisco large subunit ${ }^{31}$. Knowledge of the binding mechanism 262 now allows engineering of minimal sequence changes into native crop Rubiscos to enable binding 263 to EPYC1 and to other key proteins required to reconstitute a functional pyrenoid.

\section{Our work provides insights into pyrenoid matrix formation in other species}

266 Pyrenoids appear to have evolved independently in different lineages through convergent 267 evolution ${ }^{7,32}$. EPYC1, its Rubisco-binding sequences, and the amino acid residues that form the 268 EPYC1 binding site on the surface of Rubisco are conserved across the order Volvocales, as 269 evidenced from the genome sequences of Tetrabaena socialis, Gonium pectorale and Volvox 270 carteri (Extended Data Table 2). While the molecular mechanisms of matrix formation in other 271 lineages remain to be uncovered, candidate linker proteins have been identified based on similarity 272 of sequence properties to EPYC $1^{10}$. We hypothesize that the matrix in other lineages is formed 273 based on similar principles to those we observed in Chlamydomonas. Our experimental approach 274 for characterizing the binding interaction provides a roadmap for future structural studies of 275 pyrenoids across the tree of life.

277 This study provides a high-resolution structural view of a phase separated organelle

278 The pyrenoid matrix presents an unusual opportunity to study a two-component molecular 279 condensate where one of the components, Rubisco, is large and rigid, and the other component, 280 EPYC1, is a simple intrinsically disordered protein consisting of nearly identical tandem repeats. 281 The rigidity and size of Rubisco holoenzymes previously enabled the determination of their 
282 positions and orientations within the pyrenoid matrix of native cells by cryo-electron tomography ${ }^{5}$.

283 The identification of EPYC1 binding sites on Rubisco in the present work and the modeling of

284 linker regions between EPYC1's Rubisco binding regions now make the Chlamydomonas

285 pyrenoid matrix one of the most structurally well-defined phase separated organelles. Thus,

286 beyond advancing our structural understanding of pyrenoids, organelles that play a central role in

287 the global carbon cycle, we hope that the findings presented here will also more broadly enable

288 advances in the biophysical understanding of phase separated organelles. 


\section{Methods}

\section{Strains and culture conditions}

291 Chlamydomonas wild-type (WT) strain cMJ030 was maintained in the dark or low light $(\sim 10 \mu \mathrm{mol}$

292 photons $\mathrm{m}^{-2} \mathrm{~s}^{-1}$ ) on 1.5\% agar plates containing Tris-Acetate-Phosphate medium with revised trace

293 elements $^{33}$. For Rubisco extraction, $500 \mathrm{~mL}$ Tris-Acetate-Phosphate medium in a $1 \mathrm{~L}$ flask was

294 inoculated with a loopful of cells and the culture was grown to $4 \times 10^{6}$ cells $/ \mathrm{mL}$ at $22^{\circ} \mathrm{C}$, shaking

295 at $200 \mathrm{rpm}$ under $\sim 100 \mu \mathrm{mol}$ photons $\mathrm{m}^{-2} \mathrm{~s}^{-1}$ white light in $3 \% \mathrm{CO}_{2}$. Chlamydomonas mutant T60-

$2963^{34}$ ( $\triangle r b c s$; containing a deletion of both $R B C S$ genes) was used for generating Rubisco small

297 subunit point mutants and a wild-type control in the same background. This strain was maintained

298 on agar in the dark or low light $\left(\sim 10 \mu \mathrm{mol}\right.$ photons $\left.\mathrm{m}^{-2} \mathrm{~s}^{-1}\right)$.

\section{Protein extraction}

301 Rubisco was purified from Chlamydomonas strain cMJ030 ${ }^{35}$. Cells were disrupted by 302 ultrasonication in lysis buffer $\left(10 \mathrm{mM} \mathrm{MgCl}_{2}, 50 \mathrm{mM}\right.$ Bicine, $10 \mathrm{mM} \mathrm{NaHCO}, 1 \mathrm{mM}$ 303 dithiothreitol, $\mathrm{pH}$ 8.0) supplemented with Halt Protease Inhibitor Cocktail, EDTA-Free (Thermo

304 Fisher Scientific). The soluble lysate was fractionated by ultracentrifugation on a 10-30\% sucrose 305 gradient in a SW 41 Ti rotor at a speed of 35,000 rpm for 20 hours at $4^{\circ} \mathrm{C}$. Rubisco-containing 306 fractions were applied to an anion exchange column (MONO Q 5/50 GL, GE Healthcare) and 307 eluted with a linear salt gradient from 30 to $500 \mathrm{mM} \mathrm{NaCl}$ in lysis buffer.

\section{Peptide arrays}

310 Peptide arrays were purchased from the MIT Biopolymers Laboratory (Cambridge, MA). The 311 tiling array was composed of 18-amino-acid peptides that tiled across the full-length EPYC1 312 sequence with a step size of one amino acid. Each peptide was represented by at least two spots 313 on the array, and these replicates were averaged during data analysis. The locations of peptides on

314 the array were randomized. In the substitution arrays, peptides were designed to represent every 315 possible one-amino-acid mutation of the indicated region on EPYC1 by substitution with one of 316 the other 19 amino acids. The arrays were activated by methanol, then washed 3x10 min in binding 317 buffer (50 mM HEPES, $50 \mathrm{mM} \mathrm{KOAc,} 2 \mathrm{mM} \mathrm{Mg}(\mathrm{OAc})_{2} .4 \mathrm{H}_{2} \mathrm{O}, 1 \mathrm{mM} \mathrm{CaCl}_{2}$ and $200 \mathrm{mM}$ sorbitol, $318 \mathrm{pH}$ 6.8). The arrays were then incubated at $4^{\circ} \mathrm{C}$ with $1 \mathrm{mg}$ purified Rubisco overnight. The arrays 319 were washed in binding buffer to remove any unbound Rubisco. Using a semi-dry transfer 
320 apparatus (BIO-RAD), bound Rubisco was transferred onto an Immobilon-P PVDF membrane

321 (Millipore Sigma). Rubisco was immuno-detected with a polyclonal primary antibody raised

322 against Rubisco ${ }^{15}$ (1:10,000) followed by a HRP conjugated goat anti-rabbit (1:20,000;

323 Invitrogen). Arrays were stripped with Restore ${ }^{\mathrm{TM}}$ Western Blot Stripping Buffer before re-use

324 (Thermo Fisher Scientific).

\section{Surface plasmon resonance (SPR) experiments}

327 All the surface preparation experiments were performed at $25^{\circ} \mathrm{C}$ using a Biacore 3000 instrument

328 (GE Healthcare). Purified Rubisco was immobilized on CM5 sensor chips using a Biacore Amine 329 Coupling Kit according to the manufacturer's instructions. Briefly, the chip surface was activated 330 by an injection of $1: 1 \quad$ N-hydroxysuccinimide $\quad$ (NHS)/1-ethyl-3-(3-

331 dimethylaminopropyl)carbodiimide hydrochloride (EDC). Rubisco was diluted to $\sim 100 \mu \mathrm{g} / \mathrm{mL}$ in

$33210 \mathrm{mM}$ acetate $(\mathrm{pH} 4.5$; this $\mathrm{pH}$ had been previously optimized using the immobilization $\mathrm{pH}$ 333 scouting wizard) and was injected over the chip surface. Excess free amine groups were then 334 capped with an injection of $1 \mathrm{M}$ ethanolamine. Typical immobilization levels were 8,000 to 10,000 335 resonance units (RU), as recommended for binding experiments of small molecules. For kinetic 336 experiments (for determining the binding affinities), the typical immobilization levels were $\sim 5,000$ 337 RU. The control surfaces were prepared in exactly the same manner as the experimental surfaces 338 except that no Rubisco was injected. For immobilizations, the running buffer was the Biacore 339 HBS-EP Buffer (0.01 M HEPES pH 7.4, 0.15 M NaCl, 3 mM EDTA, 0.005\% v/v Surfactant P20).

All the binding assays were performed using the Biacore PBS-P+ Buffer $(20 \mathrm{mM}$ 341 phosphate buffer, $2.7 \mathrm{mM} \mathrm{KCl}, 137 \mathrm{mM} \mathrm{NaCl}$ and $0.05 \%$ Surfactant P20, pH 6.8) as a running 342 buffer, as recommended for small molecule analysis in Biacore systems. The analytes, consisting 343 of EPYC1 peptides synthesized by Genscript (Piscataway, New Jersey), were dissolved in the 344 same running buffer and diluted to $1 \mathrm{mM}$. The analytes were injected over the control surface and 345 experimental surfaces at a flow rate of $26 \mu \mathrm{L} / \mathrm{min}$ for 2.5 minutes, followed by 2.5 minutes of the 346 running buffer alone to allow for dissociation. The surfaces were then regenerated using running 347 buffer at a flow rate of $30 \mu \mathrm{L} / \mathrm{min}$ for 10 minutes. In all cases, binding to the control surface was 348 negligible.

349 For determining the $\mathrm{K}_{\mathrm{D}}$ of EPYC1 peptide, the kinetic assays were performed with a 350 running buffer consisting of $200 \mathrm{mM}$ sorbitol, $50 \mathrm{mM}$ HEPES, $50 \mathrm{mM}$ KOAc, $2 \mathrm{mM}$ 
$351 \mathrm{Mg}(\mathrm{OAc})_{2} .4 \mathrm{H}_{2} \mathrm{O}$ and $1 \mathrm{mM} \mathrm{CaCl}_{2}$ at $\mathrm{pH} 6.8$ (the same buffer as the peptide array assay). The

352 EPYC1 peptide was dissolved in the same running buffer as the assay and the serial dilutions were 353 also made in the same buffer. The analytes were injected over the control surface and experimental 354 surfaces at a flow rate of $15 \mu \mathrm{L} / \mathrm{min}$ for 2 minutes, followed by 10 minutes with the running buffer 355 alone to allow for dissociation. The surfaces were then regenerated by the running buffer at a flow 356 rate of $30 \mu \mathrm{L} / \mathrm{min}$ for 10 minutes. In all cases, binding to the blank chip was negligible. The fitting 357 and modeling were performed with the BIAevaluation software.

Single-particle cryo-electron microscopy data collection and image processing

360 Rubisco and peptide with the final concentration of $1.69 \mathrm{mg} / \mathrm{ml}(=3.02 \mu \mathrm{M})$ and $7.5 \mathrm{mM}$ were 361 incubated on ice for 20 minutes in buffer consisting of $200 \mathrm{mM}$ sorbitol, $50 \mathrm{mM}$ HEPES, $50 \mathrm{mM}$

$362 \mathrm{KOAc}, 2 \mathrm{mM} \mathrm{Mg}(\mathrm{OAc})_{2} .4 \mathrm{H}_{2} \mathrm{O}$ and $1 \mathrm{mM} \mathrm{CaCl}_{2}$ at $\mathrm{pH} 6.8$ (the same buffer as the peptide array 363 assay and the SPR binding assay). For both apo Rubisco and Rubisco incubated with peptide, 364 similar cryo grid-making procedures were used. 400-mesh Quantifoil 1.2/1.3 Cu grids (Quantifoil, 365 Großlöbichau, Germany) were made hydrophilic by glow discharging for 60 seconds with a 366 current of $15 \mathrm{~mA}$ in a Pelico EasiGlow system. Samples on cryo grids were plunge-frozen using 367 an FEI Mark IV Vitrobot (FEI company, part of Thermo Fisher Scientific, Hillsboro, OR). The 368 chamber of the Vitrobot was kept at $4^{\circ} \mathrm{C}$ and $100 \%$ relative humidity. $3 \mu 1$ of sample was applied 369 to the glow-discharged grid, blotted with filter paper for 3 seconds with the equipment-specific 370 blotting force set at 3 . After blotting, the grid was rapidly plunge-frozen into a liquid ethane bath. 371 Cryo grids were loaded into a $300 \mathrm{kV}$ FEI Titan Krios cryo electron microscope (FEI 372 Company) at HHMI Janelia Research Campus, Janelia Krios2, equipped with a Gatan K2 Summit 373 camera. After initial screening and evaluation, fully automated data collection was carried out 374 using SerialEM. The final exposure from each collection target was collected as a movie utilizing 375 dose fractionation on the K2 Summit camera operated in super-resolution mode. The movie was 376 collected at a calibrated magnification of $38,168 \mathrm{x}$, corresponding to $1.31 \AA$ per physical pixel in 377 the image (0.655 $\AA$ per super-resolution pixel). The dose rate on the specimen was set to be 5.82 378 electrons per $\AA^{2}$ per second and total exposure time was $10 \mathrm{~s}$, resulting in a total dose of 58.2 379 electrons per $\AA^{2}$. With dose fractionation set at $0.2 \mathrm{~s}$ per frame, each movie series contained 50 380 frames and each frame received a dose of 1.16 electrons per $\AA^{2}$. The spherical aberration constant 
of the objective lens is $2.7 \mathrm{~mm}$ and an objective aperture of $100 \mu \mathrm{m}$ was used. The nominal defocus range for the automated data collection was set to be between $-1.5 \mu \mathrm{m}$ and $-3.0 \mu \mathrm{m}$.

The movies were $2 \mathrm{x}$ binned and motion corrected using MotionCor $2^{36}$ and CTF was estimated using CTFFIND ${ }^{37}$ in Relion $3.0^{38}$. The particles were selected using cisTEM $^{39}$ and 1,809,869 peptide bound Rubisco particles and 677,071 particles in the apo state were extracted with a box size of 192x192pixels. 2D classification was performed using cisTEM 2D. The classes presenting detailed features in class averages were chosen for 3D classification on cryoSPARC ${ }^{40,41}$

388 for peptide-bound Rubisco and on Relion for the apo state. The 3D class showing clear secondary structures was chosen for 3D auto-refine first without symmetry and then with D4 symmetry imposed. After CTF refinement and Bayesian polishing in Relion, the reconstructed map resolution is $2.68 \AA$ for the apo state and $2.62 \AA$ for the peptide bound state. Details for singleparticle cryo-EM data collection and image processing are included in the Extended Data Table 1.

\section{Single-particle cryo-electron microscopy model building, fitting, and refinement}

A full model for Rubisco from Chlamydomonas was produced from an X-ray structure ${ }^{13}$ (PDB entry $1 \mathrm{GK} 8$ ) and used for rigid body fitting into a local resolution filtered cryo-EM map with an average resolution of $2.62 \AA$ using UCSF Chimera ${ }^{42}$. After rigid body fitting of the full complex, initial flexible fitting was performed in $\mathrm{COOT}^{43}$ by manually going through the entire peptide chain of a single large and small Rubisco subunit before applying the changes to the other seven large and small subunits. The sequence of the peptide was used to predict secondary structure elements using JPred $4^{44}$ which resulted in the prediction that the C-terminal region (NWRQELES)

402 is $\alpha$-helical. Guided by this prediction, the peptide was built manually into the density using 403 COOT. Additional real space refinement of the entire complex was performed using Phenix ${ }^{45}$. 404 Models were subjected to an all-atom structure validation using MolProbity ${ }^{46}$. Figures were 405 produced using UCSF Chimera.

407 Liquid-liquid phase separation assay

408 Proteins used in the liquid-liquid phase separation assay were obtained and stored essentially as 409 described previously ${ }^{11}$. Briefly, Rubisco was purified from C. reinhardtii cells (CC-2677 cw15 410 nit1-305 mt-5D, Chlamydomonas Resource Center) grown in Sueoka's high-salt medium ${ }^{47}$, using 411 a combination of anion exchange chromatography and gel filtration. 
The EPYC1 full-length gene (encoding amino acids 1-317) and corresponding R/K mutant

413 (EPYC1 ${ }^{\mathrm{R} 64 \mathrm{~A} / \mathrm{K} 127 \mathrm{~A} / \mathrm{K} 187 \mathrm{~A} / \mathrm{K} 248 \mathrm{~A} / \mathrm{R} 314 \mathrm{~A})}$ were synthesized by GenScript and cloned between the SacII

414 and HindIII site of the pHue vector ${ }^{48}$. Proteins were produced in the E. coli strain BL21 (DE3)

415 harbouring pBADESL $^{49}$ for co-expression of the E. coli chaperonin GroEL/S. The purification

416 was conducted with minor changes (dialysis for removal of high immidazol concentrations was

417 skipped by running the gel-filtration column before the second IMAC). After the first IMAC step

418 and cleavage ${ }^{50}$ of the N-terminal His 6 -ubiquitin tag, proteins were separated by gel filtration.

419 Finally, the peak fraction was passed a second time through an IMAC column, collecting EPYC1

420 from the flow through.

421 EPYC1-Rubisco condensates were reconstituted in vitro in a buffer containing $20 \mathrm{mM}$

422 Tris- $\mathrm{HCl}(\mathrm{pH} 8.0)$ and $\mathrm{NaCl}$ concentrations as indicated. $5 \mu 1$ reactions were incubated for 3 min

423 at room temperature before monitoring the droplet formation by differential interference contrast

424 (DIC) microscopy. DIC images were acquired with a Nikon Eclipse Ti Inverted Microscope using

425 a $60 \times$ oil-immersion objective after allowing the droplets to settle on the coverslip (Superior

426 Marienfeld, Germany) surface for about $3 \mathrm{~min}$. For droplet sedimentation assays $10 \mu 1$ reactions

427 were incubated for $3 \mathrm{~min}$ at $20^{\circ} \mathrm{C}$ before separating the droplets form the bulk phase by spinning

428 for $3 \mathrm{~min}$ at $21,000 \mathrm{xg}$ and $4^{\circ} \mathrm{C}$. Pelleted droplets and supernatant fractions were analyzed using

429 Coomassie-stained SDS-PAGE.

431 Yeast two-hybrid assay

432 Yeast two-hybrid to detect interactions between EPYC1 and RbcS1 was carried out as described

433 previously ${ }^{12}$. EPYC1 was cloned into the two-hybrid vector pGBKT7 to create a fusion with the

434 GAL4 DNA binding domain. Point mutations were introduced by PCR into RbcS1, which was

435 then cloned in the pGADT7 to create a fusion with the GAL4 activation domain. Yeast cells were

436 then co-transformed with binding and activation domain vectors. Successful transformants were

437 cultured, diluted to an optical density at $600 \mathrm{~nm}$ (OD600) of 0.5 or 0.1 , and plated onto SD-L-W

438 and SD-L-W-H containing increasing concentrations of the HIS3 inhibitor triaminotriazole (3-

439 AT). Plates were imaged after 3 days. Spots shown in Fig. 5a were grown at 5 mM 3-AT from a

440 starting OD600 of 0.5; they are a subset of the full dataset shown in Extended Data Fig. 6.

442 Cloning of Rubisco small subunit point mutants 
443 The plasmid pSS1-ITP ${ }^{51}$ which contains Chlamydomonas RBCS1 including UTRs and introns 1

444 and 2 was used as a starting point for generating plasmids pSH001 and pSH002, which encode

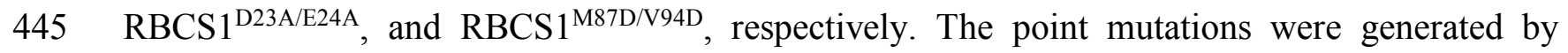

446 Gibson assembly ${ }^{52}$ of gBlocks (synthesized by Integrated DNA Technologies) containing the

447 desired mutations into pSS-ITP that had been enzyme digested by restriction endonucleases (XcmI

448 and BbvCI for the D23A/E24A mutations and BbvCI and BlpI for the M87D/V94D mutations).

449 All constructs were verified by Sanger sequencing.

450 The fragment for making pSH001 (containing the D23A/E24A Rubisco small subunit

451 mutant) had the following sequence:

452 GCAGGGCTGCCCCGGCTCAGGCCAACCAGATGATGGTCTGGACCCCGGTCAACAAC

453 AAGATGTTCGAGACCTTCTCCTACCTGCCTCCTCTGACCGCCGCGCAGATCGCCGCC

454 CAGGTCGACTACATCGTCGCCAACGGCTGGATCCCCTGCCTGGAGTTCGCTGAGGCC

455 GACAAGGCCTACGTGTCCAAC

456 The fragment for making pSH002 (containing the M87D/V94D Rubisco small subunit

457 mutant) had the following sequence:

458 CTGCCTGGAGTTCGCTGAGGCCGACAAGGCCTACGTGTCCAACGAGTCGGCCATCC

459 GCTTCGGCAGCGTGTCTTGCCTGTACTACGACAACCGCTACTGGACCATGTGGAAGC

460 TGCCCATGTTCGGCTGCCGCGACCCCGACCAGGTGCTGCGCGAGATCGACGCCTGCA

461 CCAAGGCCTTCCCCGATGCCTACGTGCGCCTGGTGGCCTTCGACAACCAGAAGCAG

462 GTGCAGATCATGGGCTTCCTGGTCCAGCGCCCCAAGACTGCCCGCGACTTCCAGCCC

463 GCCAACAAGCGCTCCGTGTAAATGGAGGCGCTCGTCGATCTGAGCCGTGTGTGATGT

464 TTGTTGGTGTTTGAGCGAGTGCAATGAGAGTGTGTGTGTGTGTGTTGTTGGTGTGTG

465 GCTAAGCCAAGCGTGATCGC

467 Transformation of Chlamydomonas to make the Rubisco small subunit point mutants

468 Chlamydomonas strains $\triangle r b c s ; R B C S^{W T}, \Delta r b c s ; R B C S^{D 23 A / E 24 A}$, and $\Delta r b c s ; R B C S^{M 87 D / V 94 D}$ were 469 generated by transforming pSS1-ITP, pSH001, and pSH002 (encoding Rubisco small subunit 470 constructs) into the Rubisco small subunit deletion mutant T60 ( $\Delta r b c s)$ by electroporation as 471 described previously ${ }^{53}$. For each transformation, $29 \mathrm{ng} \mathrm{kbp}^{-1}$ of KpnI linearized plasmid was mixed 472 with $250 \mu \mathrm{L}$ of $2 \times 10^{8}$ cells $\mathrm{mL}^{-1}$ at $16^{\circ} \mathrm{C}$ and electroporated immediately. Transformant colonies 473 were selected on Tris-Phosphate plates without antibiotics at 3\% v/v CO 2 under $\sim 50 \mu$ mol photons 
$474 \mathrm{~m}^{-2} \mathrm{~s}^{-1}$ light. The sequence of RbcS in the transformants was verified by PCR amplification and

475 Sanger sequencing.

477 Spot tests

$478 \Delta r b c s ; R B C S^{W T}, \triangle r b c s ; R B C S^{D 23 A / E 24 A}$, and $\Delta r b c s ; R B C S^{M 87 D / V 94 D}$ were grown in Tris-Phosphate

479 medium at $3 \% \mathrm{CO}_{2}$ until $\sim 2 \times 10^{6}$ cells $\mathrm{mL}^{-1}$. Cells were diluted in Tris-Phosphate medium to a 480 concentration of $8.7 \times 10^{7}$ cells $\mathrm{mL}^{-1}$, then serially diluted $1: 10$ three times. $7.5 \mu \mathrm{L}$ of each dilution 481 was spotted onto four TP plates and incubated in air or $3 \% \mathrm{CO}_{2}$ under 20 or $100 \mu \mathrm{mol}$ photons $\mathrm{m}^{-}$ $482 \quad{ }^{2} \mathrm{~s}^{-1}$ white light for 9 days before imaging.

Transmission electron microscopy

485 Samples for electron microscopy were fixed for 1 hour at room temperature in $2.5 \%$ glutaraldehyde 486 in Tris-Phosphate medium ( $\mathrm{pH} 7.4)$, followed by 1 hour at room temperature in $1 \% \mathrm{OsO}_{4}, 1.5 \%$ $487 \mathrm{~K}_{3} \mathrm{Fe}(\mathrm{CN})_{3}$, and $2 \mathrm{mM} \mathrm{CaCl}_{2}$. Fixed cells were then bulk stained for 1 hour in $2 \%$ uranyl acetate, $488 \quad 0.05 \mathrm{M}$ maleate buffer at $\mathrm{pH}$ 5.5. After serial dehydration (50\%, 75\%, 95\%, and 100\% ethanol, 489 followed by $100 \%$ acetonitrile), samples were embedded in epoxy resin containing 34\% Quetol $490651,44 \%$ nonenyl succinic anhydride, 20\% methyl-5- norbornene-2,3-dicarboxylic anhydride, and $4912 \%$ catalyst dimethylbenzylamine. Ultramicrotomy was done by the Core Imaging Lab, Medical 492 School, Rutgers University. Imaging was performed at the Imaging and Analysis Center, Princeton 493 University, on a CM100 transmission electron microscope (Philips, Netherlands) at $80 \mathrm{kV}$.

497 For detailed descriptions of the Chlamydomonas cell culture, vitrification of cells onto EM grids, 498 thinning of cells by cryo-focused ion beam milling, 3D imaging of native pyrenoids by cryo499 electron tomography, tomographic reconstruction, and subtomogram averaging, see our previous 500 study $^{5}$. In that study, we measured the distances between the center positions of Rubisco 501 complexes within tomograms of five pyrenoids. The spatial parameters determined in that study 502 were combined with the EPYC1-binding sites resolved here by cryo-EM single-particle analysis 503 to measure the nearest-neighbor distances between EPCY1-binding sites on adjacent Rubisco 504 complexes within the native pyrenoid matrix. 
The in situ subtomogram average EMD- $3694^{5}$ was used as the reference for the Rubisco model. We extracted the isosurface from this density using the 0.5 contour level recommended in

507 the Electron Microscopy Data Bank entry. We then fit the atomic model of EPYC1-bound Rubisco 508 (Fig. 2) within the EMD-3694 density, and for each EPYC1-binding site, we marked the closest point on the isosurface to define the EPYC1 binding sites on this model. The positions and orientations previously determined by subtomogram averaging were used to place each Rubisco model and its corresponding binding sites into the pyrenoid tomograms using the PySeg program ${ }^{54}$.

513 Rubisco complexes, first, linkers were drawn between each EPYC1 binding site and all other 514 binding sites within $25 \mathrm{~nm}$. Binding sites on the same Rubisco complex were ignored. Next, the 515 linkers were filtered by length (defined as the Euclidean distance between the two binding sites), 516 and only the shortest linker was retained for each binding site. To prevent edge effects, linkers 517 were discarded if they had a binding site $<12 \mathrm{~nm}$ from the masked excluded volume (grey in Fig. 518 6b), which marks the border of the analyzed pyrenoid matrix. Finally, linker distances were plotted in a histogram to show the distribution of lengths (normalized to $100 \%$ ).

\section{Modeling of the energy required to stretch EPYC1-linker regions}

522 The energy required to stretch the linker regions between EPYC1's Rubisco-binding regions was 523 determined as follows. The force $\mathrm{F}$ required to stretch a 40 amino acid linker region to any given 524 length $\mathrm{z}$ was approximated using a wormlike chain model ${ }^{55}$ :

$$
F(z)=\frac{k_{\mathrm{B}} T}{4 L_{p}}\left[\frac{1}{\left(1-z / L_{0}\right)^{2}}-1+\frac{4 z}{L_{0}}\right]
$$

In the above equation, $k_{\mathrm{B}}$ is the Boltzmann constant, $T$ is the temperature, $\mathrm{L}_{\mathrm{p}}$ is the persistence

527 length (assumed to be $1 \mathrm{~nm}$, a representative value for disordered proteins), and $\mathrm{L}_{0}$ is the contour 528 length (estimated as 40 amino acids * $0.36 \mathrm{~nm} /$ amino acid). The energy required to stretch the 529 linker to a length $\mathrm{x}$ is given by:

$$
E(x)=\int_{0}^{x} F(z) d z
$$

531 This energy was calculated and plotted in Fig. 6d. 


\section{References}

5341 Field, C. B., Behrenfeld, M. J., Randerson, J. T. \& Falkowski, P. Primary production of the biosphere: integrating terrestrial and oceanic components. Science 281, 237-240 (1998).

5362 Hessler, A. M., Lowe, D. R., Jones, R. L. \& Bird, D. K. A lower limit for atmospheric carbon

3 Ainsworth, E. A. \& Long, S. P. What have we learned from 15 years of free-air CO2 enrichment (FACE)? A meta-analytic review of the responses of photosynthesis, canopy properties and plant production to rising CO2. New Phytol 165, 351-371, doi:10.1111/j.14698137.2004.01224.x (2005).

4 Raven, J. A., Cockell, C. S. \& De La Rocha, C. L. The evolution of inorganic carbon concentrating mechanisms in photosynthesis. Philosophical transactions of the Royal Society of London. Series B, Biological sciences 363, 2641-2650, doi:10.1098/rstb.2008.0020 (2008).

5 Freeman Rosenzweig, E. S. et al. The Eukaryotic CO2-Concentrating Organelle Is Liquid-

6 Badger, M. R. et al. The diversity and coevolution of Rubisco, plastids, pyrenoids, and like and Exhibits Dynamic Reorganization. Cell 171, 148-162 e119, doi:10.1016/j.cell.2017.08.008 (2017).

8 Wang, Y., Stessman, D. J. \& Spalding, M. H. The CO2 concentrating mechanism and

9 Raven, J. A. CO2-concentrating mechanisms: A direct role for thylakoid lumen acidification? chloroplast-based CO2-concentrating mechanisms in algae. Can J Bot 76, 1052-1071, doi:10.1139/b98-074 (1998).

10 Mackinder, L. C. et al. A repeat protein links Rubisco to form the eukaryotic carbonphotosynthetic carbon assimilation in limiting CO2 : how Chlamydomonas works against the gradient. The Plant journal : for cell and molecular biology 82, 429-448, doi:10.1111/tpj.12829 (2015). concentrating organelle. Proc Natl Acad Sci $U$ S A 113, 5958-5963, doi:10.1073/pnas.1522866113 (2016).

11 Wunder, T., Cheng, S. L. H., Lai, S. K., Li, H. Y. \& Mueller-Cajar, O. The phase separation underlying the pyrenoid-based microalgal Rubisco supercharger. Nature communications $\mathbf{9}$, 5076, doi:10.1038/s41467-018-07624-w (2018). 
12 Atkinson, N. et al. The pyrenoidal linker protein EPYC1 phase separates with hybrid Arabidopsis-Chlamydomonas Rubisco through interactions with the algal Rubisco small subunit. Journal of experimental botany 70, 5271-5285, doi:10.1093/jxb/erz275 (2019).

13 Taylor, T. C., Backlund, A., Bjorhall, K., Spreitzer, R. J. \& Andersson, I. First crystal structure of Rubisco from a green alga, Chlamydomonas reinhardtii. J Biol Chem 276, 48159-48164,

14 Duff, A. P., Andrews, T. J. \& Curmi, P. M. The transition between the open and closed states of rubisco is triggered by the inter-phosphate distance of the bound bisphosphate. $J$ Mol Biol 298, 903-916, doi:10.1006/jmbi.2000.3724 (2000).

15 Meyer, M. T. et al. Rubisco small-subunit $\alpha$-helices control pyrenoid formation in Chlamydomonas. Proc Natl Acad Sci $U$ S A 109, 19474-19479, doi:10.1073/pnas.1210993109. (2012).

16 Engel, B. D. et al. Native architecture of the Chlamydomonas chloroplast revealed by in situ cryo-electron tomography. eLife 4, DOI: 10.7554/eLife.04889, doi:10.7554/eLife.04889 (2015).

17 Goodenough, U. W. \& Levine, R. P. Chloroplast structure and function in ac-20, a mutant strain of Chlamydomonas reinhardi. 3. Chloroplast ribosomes and membrane organization. $J$

18 Ma, Y., Pollock, S. V., Xiao, Y., Cunnusamy, K. \& Moroney, J. V. Identification of a novel gene, CIA6, required for normal pyrenoid formation in Chlamydomonas reinhardtii. Plant Physiol 156, 884-896, doi:10.1104/pp.111.173922 (2011).

19 Caspari, O. D. et al. Pyrenoid loss in Chlamydomonas reinhardtii causes limitations in CO2 supply, but not thylakoid operating efficiency. Journal of experimental botany 68, 3903-3913, doi:10.1093/jxb/erx197 (2017).

$20 \mathrm{Li}$, P. et al. Phase transitions in the assembly of multivalent signalling proteins. Nature 483, 336-340, doi:10.1038/nature10879 (2012).

21 Borkhsenious, O. N., Mason, C. B. \& Moroney, J. V. The Intracellular Localization of Ribulose-1,5-Bisphosphate Carboxylase/Oxygenase in Chlamydomonas reinhardtii. Plant Physiol 116, 1585-1591, doi:10.1104/pp.116.4.1585 (1998).

22 Turkina, M. V., Blanco-Rivero, A., Vainonen, J. P., Vener, A. V. \& Villarejo, A. CO2 limitation induces specific redox-dependent protein phosphorylation in Chlamydomonas reinhardtii. Proteomics 6, 2693-2704, doi:10.1002/pmic.200500461 (2006).

23 Cai, F. et al. Advances in Understanding Carboxysome Assembly in Prochlorococcus and doi:10.3390/life5021141 (2015). 
24 Oltrogge, L. M. et al. Multivalent interactions between CsoS2 and Rubisco mediate alphacarboxysome formation. Nat Struct Mol Biol 27, 281-287, doi:10.1038/s41594-020-0387-7 (2020).

25 Long, B. M., Badger, M. R., Whitney, S. M. \& Price, G. D. Analysis of carboxysomes from Synechococcus PCC7942 reveals multiple Rubisco complexes with carboxysomal proteins CcmM and CcaA. J Biol Chem 282, 29323-29335, doi:10.1074/jbc.M703896200 (2007).

26 Wang, H. et al. Rubisco condensate formation by $\mathrm{CcmM}$ in beta-carboxysome biogenesis. Nature 566, 131-135, doi:10.1038/s41586-019-0880-5 (2019).

27 Hennacy, J. H. \& Jonikas, M. C. Prospects for Engineering Biophysical CO2 Concentrating Mechanisms into Land Plants to Enhance Yields. Annual review of plant biology, doi:10.1146/annurev-arplant-081519-040100 (2020).

28 Long, B. M. et al. Carboxysome encapsulation of the CO2-fixing enzyme Rubisco in tobacco chloroplasts. Nature communications 9, 3570, doi:10.1038/s41467-018-06044-0 (2018).

29 Lin, M. T., Occhialini, A., Andralojc, P. J., Parry, M. A. \& Hanson, M. R. A faster Rubisco with potential to increase photosynthesis in crops. Nature 513, 547-550, doi:10.1038/nature13776 (2014).

30 Atkinson, N. et al. Introducing an algal carbon-concentrating mechanism into higher plants: location and incorporation of key components. Plant Biotechnol J 14, 1302-1315, doi:10.1111/pbi.12497 (2016).

31 Hanson, M. R., Gray, B. N. \& Ahner, B. A. Chloroplast transformation for engineering of photosynthesis. Journal of experimental botany 64, 731-742, doi:10.1093/jxb/ers325 (2013).

32 Raven, J. A., Beardall, J. \& Sanchez-Baracaldo, P. The possible evolution and future of CO2concentrating mechanisms. J Exp Bot 68, 3701-3716, doi:10.1093/jxb/erx110 (2017).

33 Kropat, J. et al. A revised mineral nutrient supplement increases biomass and growth rate in Chlamydomonas reinhardtii. The Plant journal : for cell and molecular biology 66, 770-780, doi:10.1111/j.1365-313X.2011.04537.x (2011).

34 Khrebtukova, I. \& Spreitzer, R. J. Elimination of the Chlamydomonas gene family that encodes the small subunit of ribulose-1,5-bisphosphate carboxylase/oxygenase. Proc Natl Acad Sci U S A 93, 13689-13693, doi:10.1073/pnas.93.24.13689 (1996).

35 Zhang, R. et al. High-Throughput Genotyping of Green Algal Mutants Reveals Random Distribution of Mutagenic Insertion Sites and Endonucleolytic Cleavage of Transforming DNA. Plant Cell 26, 1398-1409, doi:10.1105/tpc.114.124099 (2014).

36 Zheng, S. Q. et al. MotionCor2: anisotropic correction of beam-induced motion for improved cryo-electron microscopy. Nat Methods 14, 331-332, doi:10.1038/nmeth.4193 (2017). 
37 Rohou, A. \& Grigorieff, N. CTFFIND4: Fast and accurate defocus estimation from electron micrographs. J Struct Biol 192, 216-221, doi:10.1016/j.jsb.2015.08.008 (2015).

38 Zivanov, J. et al. New tools for automated high-resolution cryo-EM structure determination in RELION-3. eLife 7, doi:10.7554/eLife.42166 (2018).

39 Grant, T., Rohou, A. \& Grigorieff, N. cisTEM, user-friendly software for single-particle image processing. eLife 7, doi:10.7554/eLife.35383 (2018).

40 Punjani, A., Rubinstein, J. L., Fleet, D. J. \& Brubaker, M. A. cryoSPARC: algorithms for rapid unsupervised cryo-EM structure determination. Nat Methods 14, 290-296, doi:10.1038/nmeth.4169 (2017).

41 Punjani, A., Brubaker, M. A. \& Fleet, D. J. Building Proteins in a Day: Efficient 3D Molecular Structure Estimation with Electron Cryomicroscopy. IEEE Trans Pattern Anal Mach Intell 39, 706-718, doi:10.1109/TPAMI.2016.2627573 (2017).

42 Pettersen, E. F. et al. UCSF Chimera--a visualization system for exploratory research and analysis. J Comput Chem 25, 1605-1612, doi:10.1002/jcc.20084 (2004).

43 Emsley, P., Lohkamp, B., Scott, W. G. \& Cowtan, K. Features and development of Coot. Acta Crystallogr D Biol Crystallogr 66, 486-501, doi:10.1107/S0907444910007493 (2010).

44 Drozdetskiy, A., Cole, C., Procter, J. \& Barton, G. J. JPred4: a protein secondary structure

45 Adams, P. D. et al. PHENIX: a comprehensive Python-based system for macromolecular structure solution. Acta Crystallogr D Biol Crystallogr 66, 213-221, doi:10.1107/S0907444909052925 (2010).

46 Chen, V. B. et al. MolProbity: all-atom structure validation for macromolecular crystallography. Acta Crystallogr
doi:10.1107/S0907444909042073 (2010).

47 Sueoka, N. Mitotic replication of deoxyribonucleic acid in Chlamydomonas reinhardi. Proc Natl Acad Sci U S A. 46, 83-91, doi:10.1073/pnas.46.1.83 (1960). system for high-level expression and easy purification of authentic recombinant proteins.

49 Ewalt, K. L., Hendrick, J. P., Houry, W. A. \& Hartl, F. U. In vivo observation of polypeptide flux through the bacterial chaperonin system. Cell 90, 491-500, doi:10.1016/s00928674(00)80509-7 (1997).

50 Baker, R. T. et al. in Ubiquitin and Protein Degradation, Part A Methods in Enzymology 540-554 (2005). 
51 Genkov, T., Meyer, M., Griffiths, H. \& Spreitzer, R. J. Functional hybrid rubisco enzymes with plant small subunits and algal large subunits: engineered rbcS cDNA for expression in chlamydomonas. J Biol Chem 285, 19833-19841, doi:10.1074/jbc.M110.124230 (2010).

52 Gibson, D. G. et al. Enzymatic assembly of DNA molecules up to several hundred kilobases. Nature methods 6, 343-345, doi:10.1038/nmeth.1318 (2009).

$53 \mathrm{Li}, \mathrm{X}$. et al. A genome-wide algal mutant library and functional screen identifies genes required for eukaryotic photosynthesis. Nat Genet 51, 627-635, doi:10.1038/s41588-0190370-6 (2019).

54 Martinez-Sanchez, A. et al. Template-free detection and classification of membrane-bound complexes in cryo-electron tomograms. Nat Methods, doi:10.1038/s41592-019-0675-5 (2020).

55 Cheng, S., Cetinkaya, M. \& Grater, F. How sequence determines elasticity of disordered proteins. Biophys J 99, 3863-3869, doi:10.1016/j.bpj.2010.10.011 (2010).

\section{Acknowledgements}

We thank Jianping Wu, Nieng Yan, Luke Mackinder, Cliff Brangwynne and members of the Jonikas laboratory for helpful discussions; Ned Wingreen, Silvia Ramundo, Jessi Hennacy, and Eric Franklin for constructive feedback on the manuscript; Wolfgang Baumeister and Jürgen Plitzko for providing support and cryo-ET instrumentation; and Miroslava Schaffer for help with acquiring the cryo-ET data, previously published in Freeman Rosenzweig et al., 2017. This project was funded by National Science Foundation (IOS-1359682 and MCB-1935444), National Institutes of Health (DP2-GM-119137), and Simons Foundation and Howard Hughes Medical Institute (55108535) grants to M.C.J., Deutsche Forschungsgemeinschaft grant (EN 1194/1-1 as part of FOR2092) to B.D.E., Ministry of Education (MOE Singapore) Tier 2 grant (MOE2018T2-2-059) to O.M.-C., UK Biotechnology and Biological Sciences Research Council (BB/S015531/1) and Leverhulme Trust (RPG-2017-402) grants to A.J.M and N.A., NIH grant R01GM071574 to F.M.H., Deutsche Forschungsgemeinschaft fellowship (PO2195/1-1) to S.A.P., and National Institute of General Medical Sciences of the National Institutes of Health (T32GM007276) training grant to V.K.C.. The content is solely the responsibility of the authors and does not necessarily represent the official view of the National Institutes of Health. 


\section{Author contributions}

703 S.H., P.D.J., V.C., F.M.H., T.W., O.M.-C., B.D.E., and M.C.J. designed experiments. S.H. 704 identified EPYC1's Rubisco-binding regions on EPYC1 by peptide tiling array and SPR. S.H. and

705 S.A.P. prepared the Rubisco and EPYC1 peptide sample for single-particle cryo-EM; S.H., S.A.P. 706 and G.H. prepared the Rubisco samples for peptide tiling array and surface plasmon resonance.

707 H.-T.C., D.M. and Z.Y. performed Cryo-EM grid preparation, sample screening, data acquisition, 708 image processing, reconstruction and map generation. D.M. and P.D.J. carried out single-particle 709 model building and fitting and refinement. S.H., H.-T.C., D.M., P.D.J., F.M.H. and M.C.J. 710 analyzed the structures. S.H. and W.P. analyzed EPYC1 binding to Rubisco by peptide substitution

711 array and SPR. T.W. performed in vitro reconstitution phase separation experiments. N.A. and 712 A.J.M. performed yeast two-hybrid experiments. S.H. and M.T.M. made Rubisco small subunit 713 point mutants. S.H. performed spot test experiments. M.T.M. performed TEM. A.M.-S. performed 714 the cryo-ET data analysis and modeling. S.H. and M.C.J. wrote the manuscript. All authors read 715 and commented on the manuscript.

716

\section{Conflict of interest statement}

718 Princeton University and HHMI have submitted a provisional patent application on aspects of 719 the findings. 

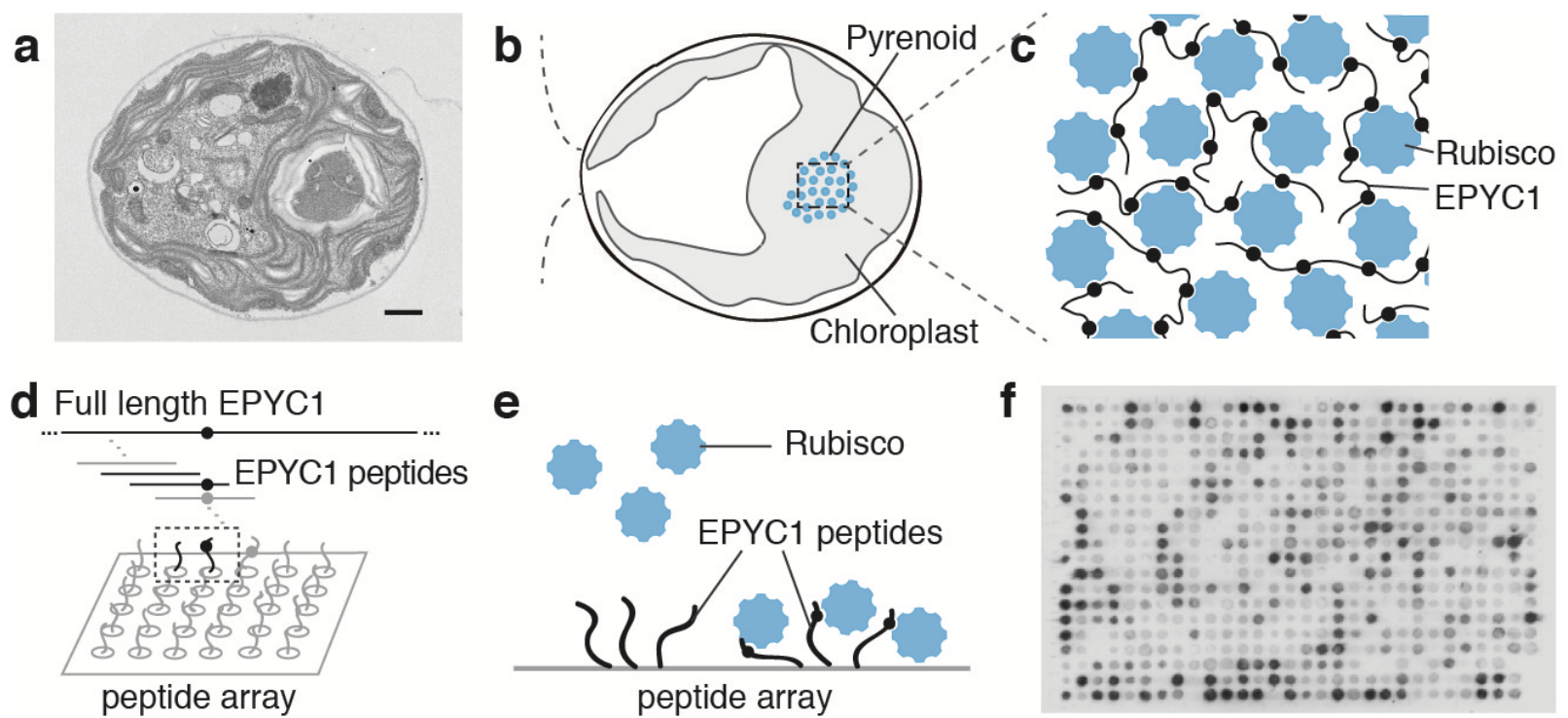

g

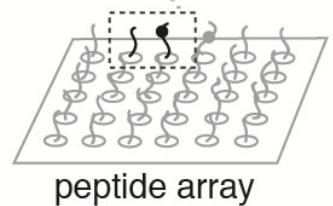

peptide array

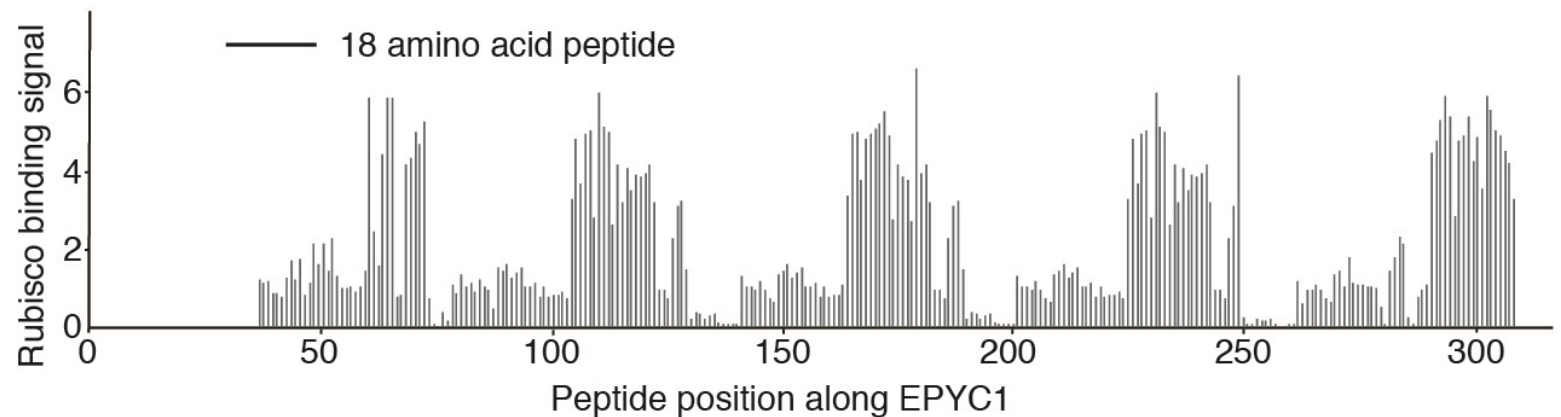

h

\begin{tabular}{|r|r|r|r|r|r|r}
\multicolumn{1}{c}{ cTP } & Repeat 1 & Repeat 2 & Repeat 3 & Repeat 4 & Repeat 5 \\
\hline & $m$ & $m$ & $m$ & $m$ & $v$ \\
\hline
\end{tabular}

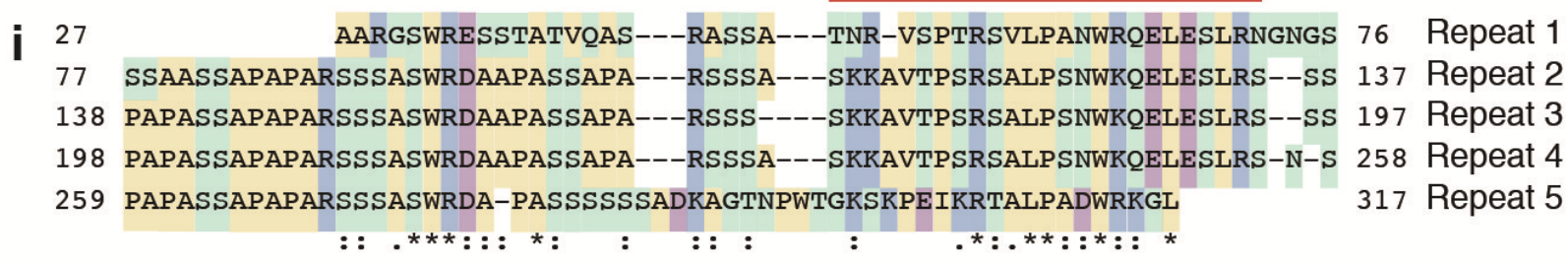

\begin{tabular}{l|l|l} 
Nonpolar and hydrophobic & Polar and hydrophilic & Basic
\end{tabular}

724 Fig. 1 | EPYC1 consists of five tandem sequence repeats, each of which contains a Rubisco-

725 binding region. a, Transmission electron microscopy (TEM) image of a Chlamydomonas cell.

726 Scale bar $=1 \mu \mathrm{m}$. $\mathbf{b}$, Cartoon depicting the chloroplast and pyrenoid in the image shown in panel

727 a. The blue dots indicate the location of Rubisco enzymes clustered in the pyrenoid matrix. c, We 
728 hypothesized that pyrenoid matrix formation is mediated by multivalent interactions between

729 Rubisco and the intrinsically disordered protein EPYC1. d, We designed an array of 18 amino acid

730 peptides tiling across the full length EPYC1 sequence. e, Incubation of the array with purified

731 Rubisco allows identification of peptides that bind to Rubisco. f, Image of the Rubisco binding

732 signal from the peptide tiling array. g, The Rubisco binding signal was quantified and plotted for

733 each peptide as a function of the position of the middle of the peptide along the EPYC1 sequence.

734 The initial 26 amino acids of EPYC1 correspond to a chloroplast targeting peptide (cTP), which

735 is not present in the mature protein ${ }^{12}$. Results are representative of three independent experiments.

$736 \mathbf{h}$, The positions of EPYC1's five sequence repeats are shown to scale with panel g. Predicted $\alpha$ -

737 helical regions are shown as wavy lines. i, Primary sequence of EPYC1, with the five sequence

738 repeats aligned. In panels $\mathrm{h}$ and $\mathrm{i}$, the region used for structural studies $\left(\mathrm{EPYC} 1_{49-72)}\right.$ is indicated

739 by a red line. 

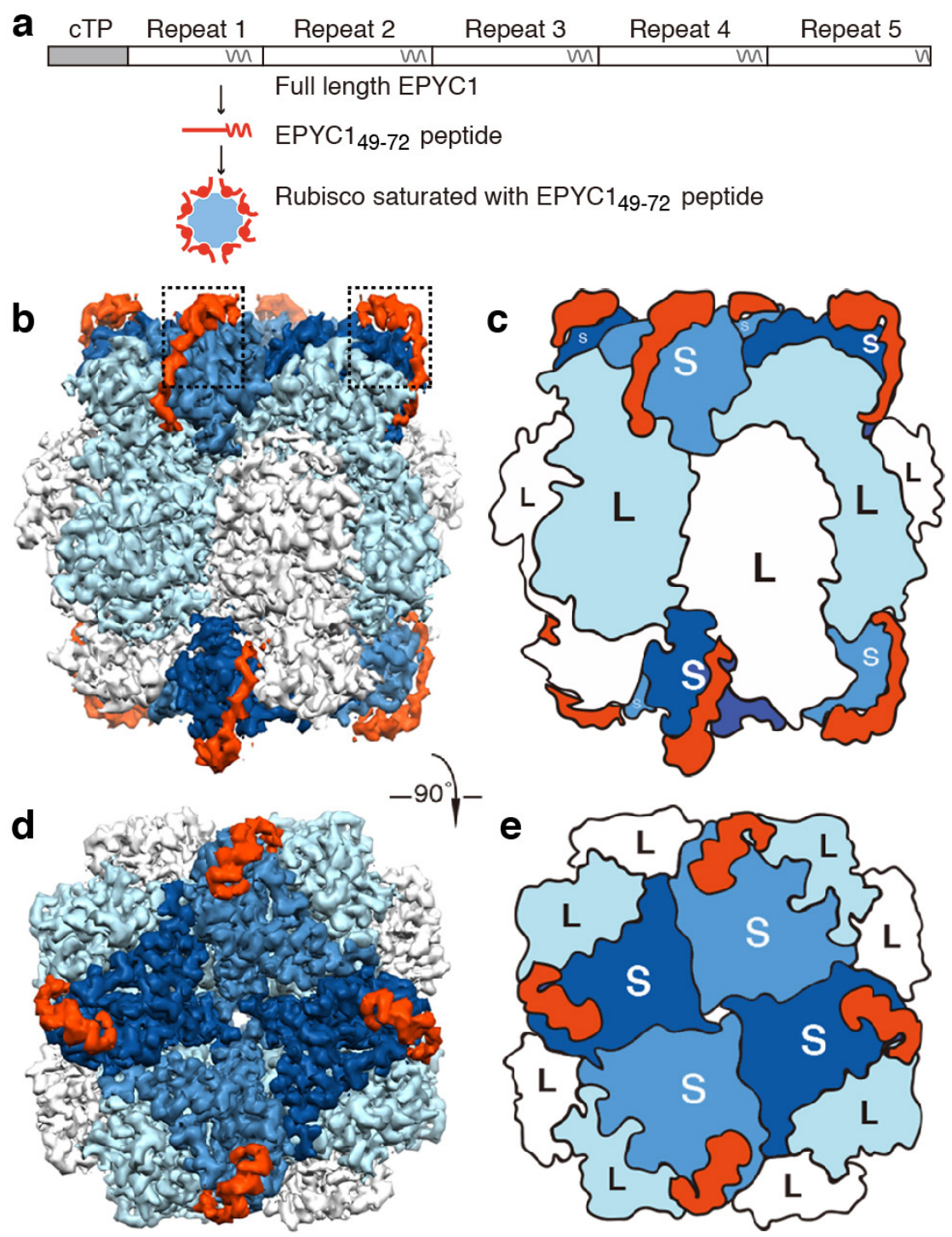

Rubisco small subunit

EPYC1 peptide

742 Fig. 2 | EPYC1 binds to Rubisco small subunits. a, Peptide EPYC149-72, corresponding to the

743 first Rubisco-binding region of EPYC1, was incubated at saturating concentrations with Rubisco

744 prior to single particle cryo-electron microscopy. b-e, Density maps (b, d) and cartoons (c, e)

745 illustrate the side views (b, c) and top views (d, e) of the density map of the EPYC1 peptide-

746 Rubisco complex. Dashes in panel b indicate regions shown in Fig.3a-3f. 

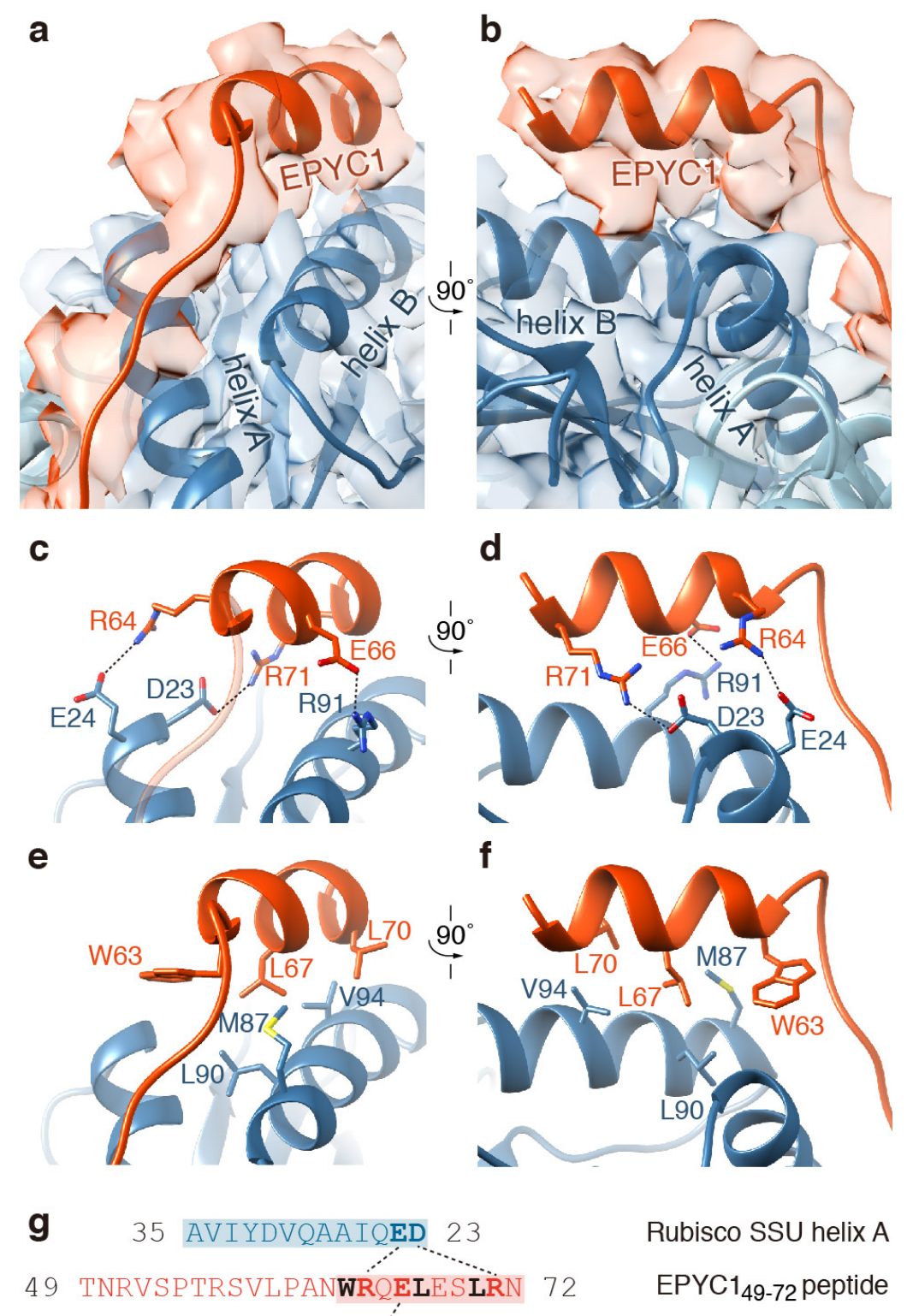

Rubisco SSU helix A

Fig. 3 | EPYC1 binds to Rubisco small subunit $\alpha$-helices via salt bridges and a hydrophobic pocket. a-b, Front (a) and side (b) views of the EPYC1 peptide (red) bound to the two $\alpha$-helices of the Rubisco small subunit (blue). c-d, Three pairs of residues form salt bridges between the helix of the EPYC1 peptide and the helices on the Rubisco small subunit. Shown are front (c) and

754 side (d) views as in panel a and panel b. The distances from EPYC1 R64, R71 and E66 to Rubisco 755 small subunit E24, D23 and R91 are $3.06 \AA$, $3.23 \AA$, and $3.13 \AA$, respectively. e-f, A hydrophobic 756 pocket is formed by three residues of the EPYC1 peptide and three residues of helix B of the 757 Rubisco small subunit. Shown are front (e) and side (f) views as in panel a and panel b. g, Summary 
758 of the interactions observed between the EPYC1 peptide and the two $\alpha$-helices of the Rubisco

759 small subunit. Helices are highlighted; the residues mediating interactions are bold; salt bridges

760 are shown as dotted lines; residues contributing to the hydrophobic pocket are shown in black. 


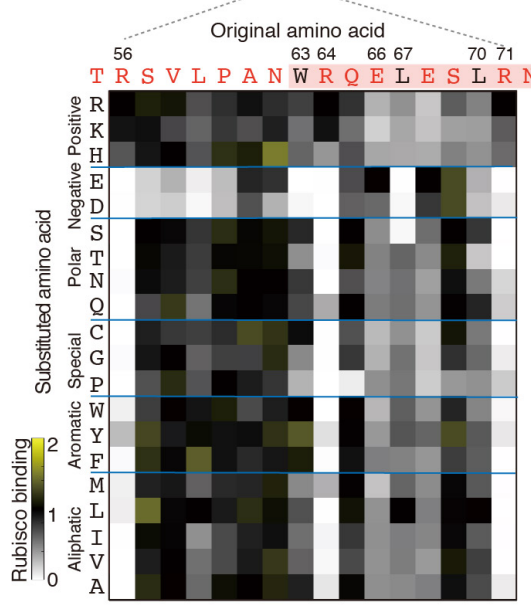

b

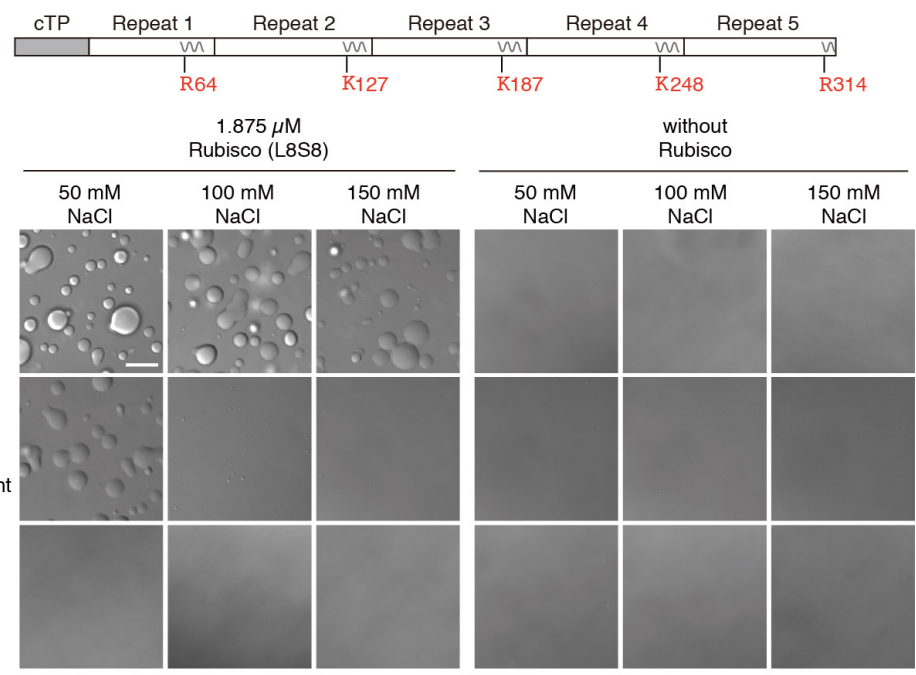

763 Fig. 4 | Interface residues on EPYC1 are required for binding and phase separation of

764 EPYC1 and Rubisco in vitro. a, Rubisco binding to a peptide array representing every possible

765 single amino acid substitution for amino acids 56-71 of EPYC1. The binding signal was

766 normalized by the binding signal of the original sequence. $\mathbf{b}$, The effect of mutating the central $\mathrm{R}$

767 or K in each of EPYC1's Rubisco-binding regions on in vitro phase separation of EPYC1 with

768 Rubisco. Scale bar $=10 \mu \mathrm{m}$. 
a

Rubisco SSU EPYC1 Control Test

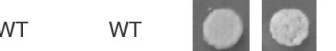

D23A WT

E24A WT

R91A WT

V94D b $\frac{1}{2}$

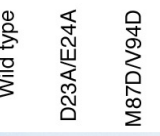

言

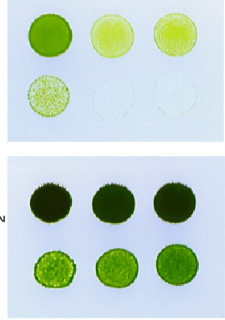

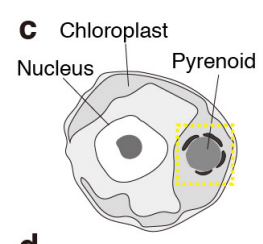

d

Starch Matrix

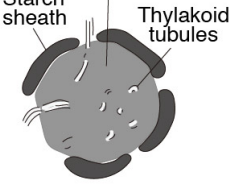

Wild type

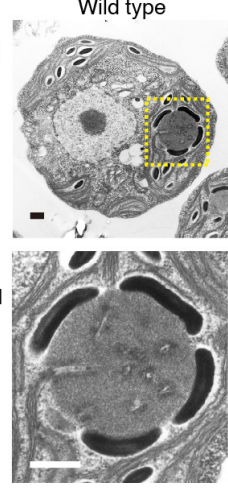

D23A/E24A

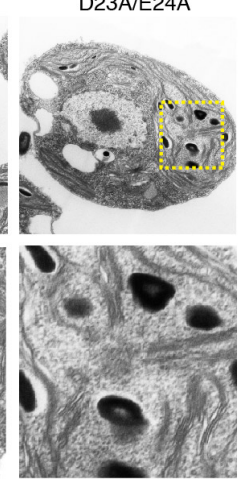

M87D/V94D

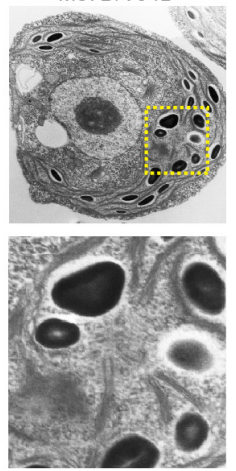

Fig. 5 | Interface residues on Rubisco are required for Yeast Two-Hybrid interactions

772 between EPYC1 and Rubisco, and for pyrenoid matrix formation in vivo. a, The importance

773 of Rubisco small subunit residues for interaction with EPYC1 was tested by mutagenesis in a yeast

774 two-hybrid experiment. b. The Rubisco small subunit-less mutant T60 ( $\Delta r b c s)$ was transformed

775 with wild-type, D23A/E24A or M87D/V94D Rubisco small subunits. Serial 1:10 dilutions of cell

776 cultures were spotted on TP minimal medium and grown in air or $3 \% \mathrm{CO}_{2}$. c-d, Representative

777 electron micrographs of the whole cells (c) and corresponding pyrenoids (d) of the strains

778 expressing wild-type, D23A/E24A, and M87D/V94D Rubisco small subunit. Dashes in panel c indicate regions shown in panel F. Scale bars $=500 \mathrm{~nm}$. 

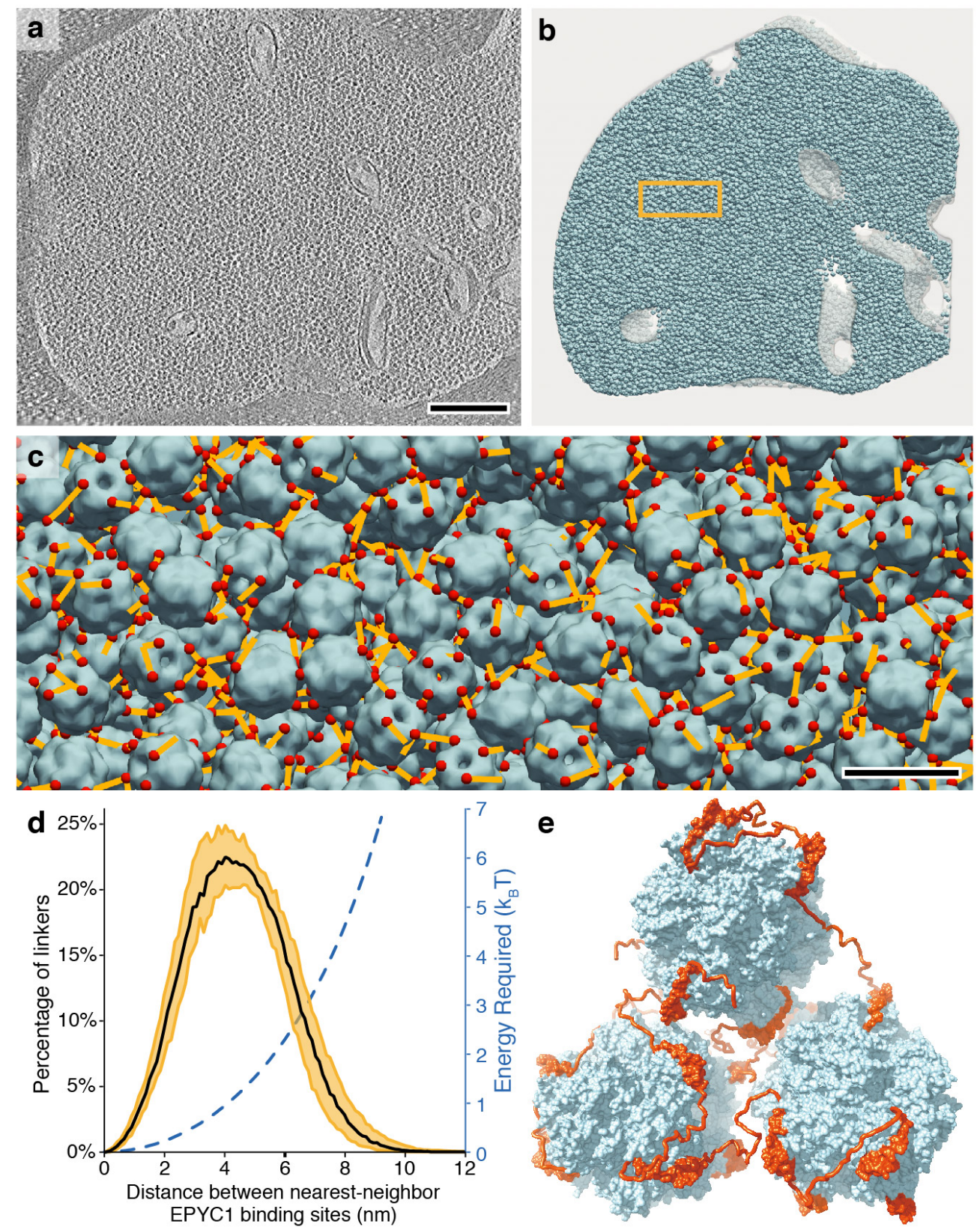

782 Fig. 6 | A model for matrix structure consistent with in situ Rubisco positions and

783 orientations. a, The pyrenoid matrix was imaged by cryo-electron tomography ${ }^{5}$. An individual

784 slice through the three-dimensional volume is shown. Scale bar $=200 \mathrm{~nm}$. b, The positions and

785 orientations of individual Rubisco holoenzymes (blue) were determined by subtomogram

786 averaging and fit into the tomogram volume. c, The distances (yellow) between the nearest

787 EPYC1-binding sites (red) on neighboring Rubisco holoenzymes (blue) were measured. The view

788 is from inside the matrix; in some cases the nearest EPYC1 binding site is on a Rubisco that is out 
789 of the field of view, causing some yellow lines to appear unconnected in this image. Scale bar $=$

$79020 \mathrm{~nm}$. d, Histogram showing the distances between the nearest EPYC1 binding sites on

791 neighboring Rubisco holoenzymes. Shading indicates 95\% confidence interval based on data from

792 five independent tomograms. The estimated energy required for stretching a chain of 40 amino

793 acids a given distance is shown in blue. e, A 3D model illustrates how EPYC1 (red) could crosslink

794 multiple Rubisco holoenzymes (blue) to form the pyrenoid matrix. The conformations of the

795 intrinsically disordered linkers between EPYC1 binding sites were modeled hypothetically. 

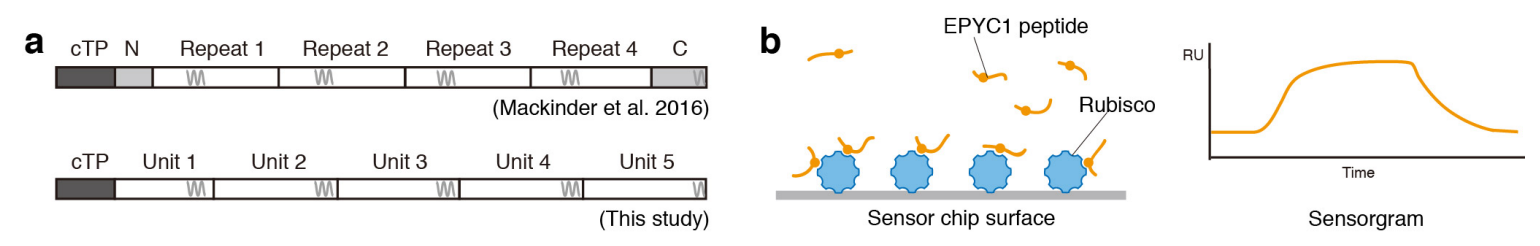

C
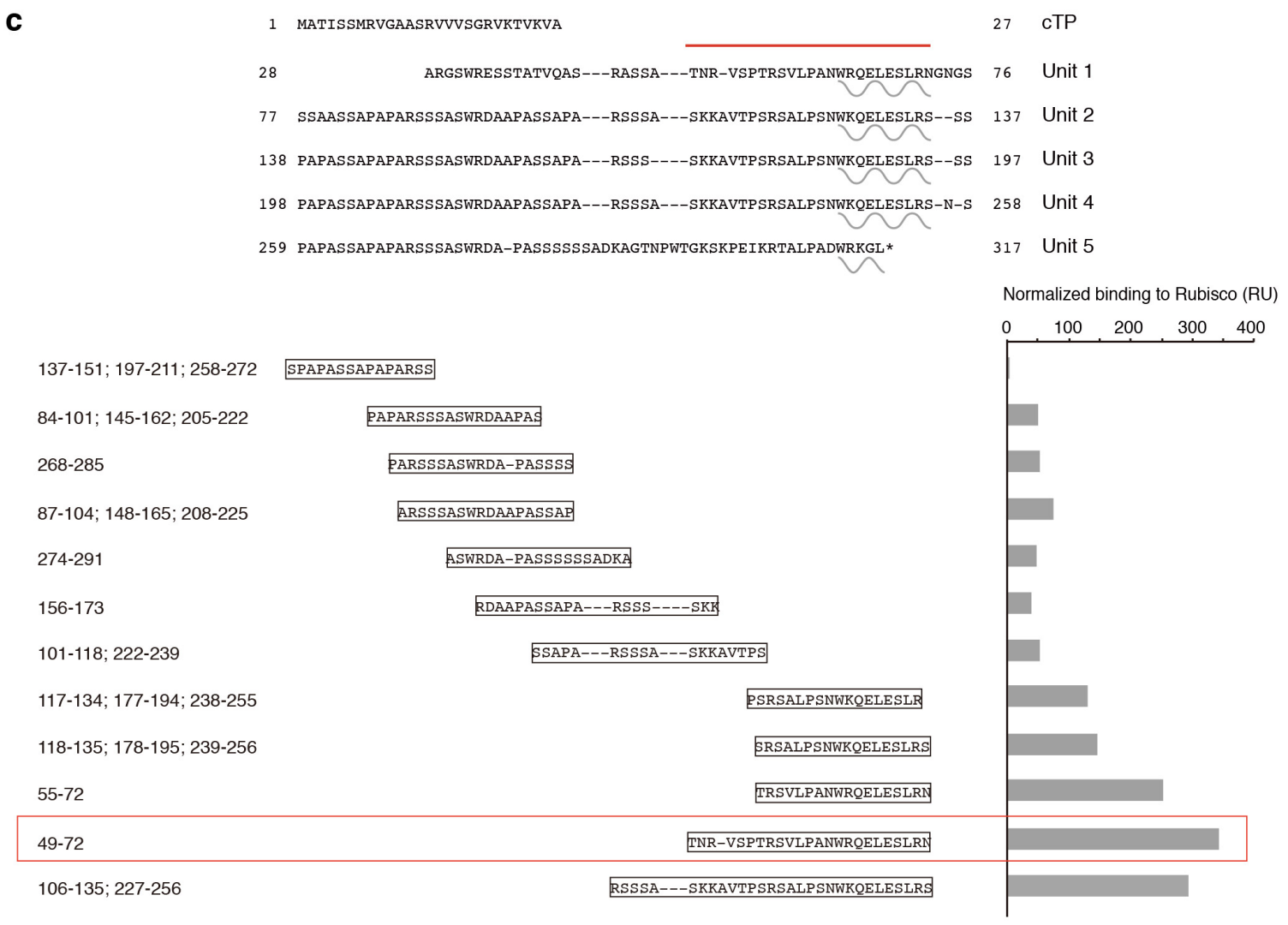

d

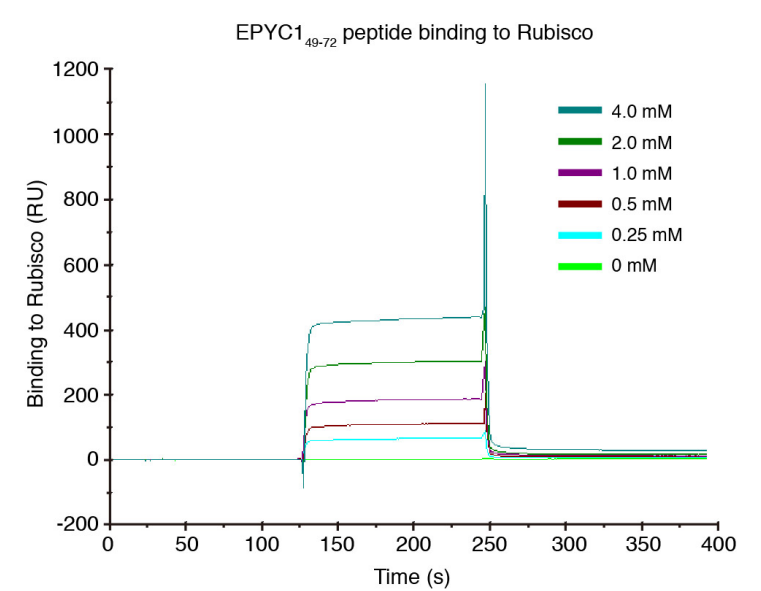

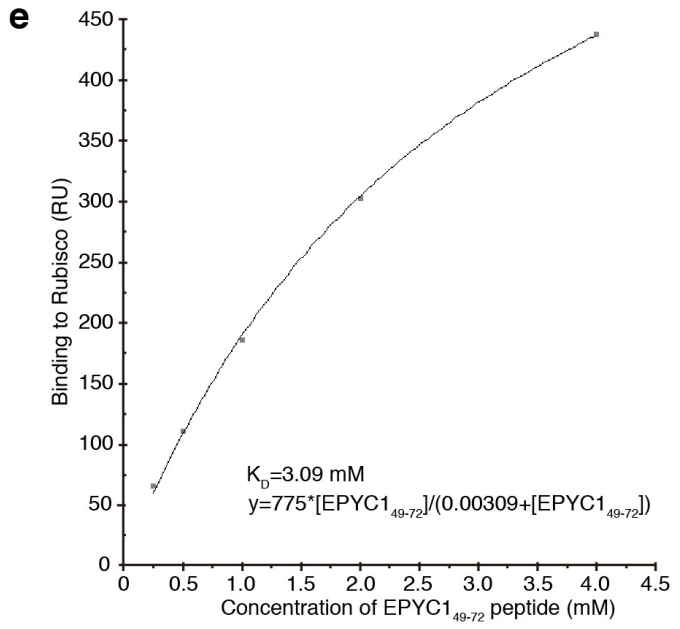

Extended Data Fig. 1 | The EPYC1 peptide with the highest binding affinity to Rubisco was

798 chosen for structural studies. a, Diagram indicating the differences between the previously 799 defined sequence repeats ${ }^{10}$ and the newly defined sequence repeats on full-length EPYC1. b, To 
800 verify the Rubisco-binding regions on EPYC1, surface plasmon resonance (SPR) was used to 801 measure the binding of EPYC1 peptides to Rubisco. Purified Rubisco was immobilized on a sensor

802 surface, and the EPYC1 peptides in solution were injected over the surface. The binding activity 803 was recorded in real time in a sensorgram. c, The peptides used in SPR experiments are shown 804 aligned to the sequence as shown in Fig. 1. The Rubisco-binding signal from the SPR experiment 805 of each peptide is shown after normalization to the peptide's molecular weight. EPYC149-72 was 806 chosen for structural studies based on its reproducible high Rubisco binding signal. d, The 807 Rubisco-binding response of the EPYC1 $49-72$ peptide at different concentrations was measured by 808 SPR. e, The binding responses shown in (d) were fitted to estimate the $\mathrm{K}_{\mathrm{D}}$ of EPYC1 ${ }_{49-72}$ peptide 809 binding to Rubisco. 
bioRxiv preprint doi: https://doi.org/10.1101/2020.08.16.252809; this version posted August 16. 2020. The copyright holder for this preprint (which was not certified by peer review) is the author/funder, who has granted bioRxiv a license to display the preprint in perpetuity. It is made available under aCC-BY-NC-ND 4.0 International license.
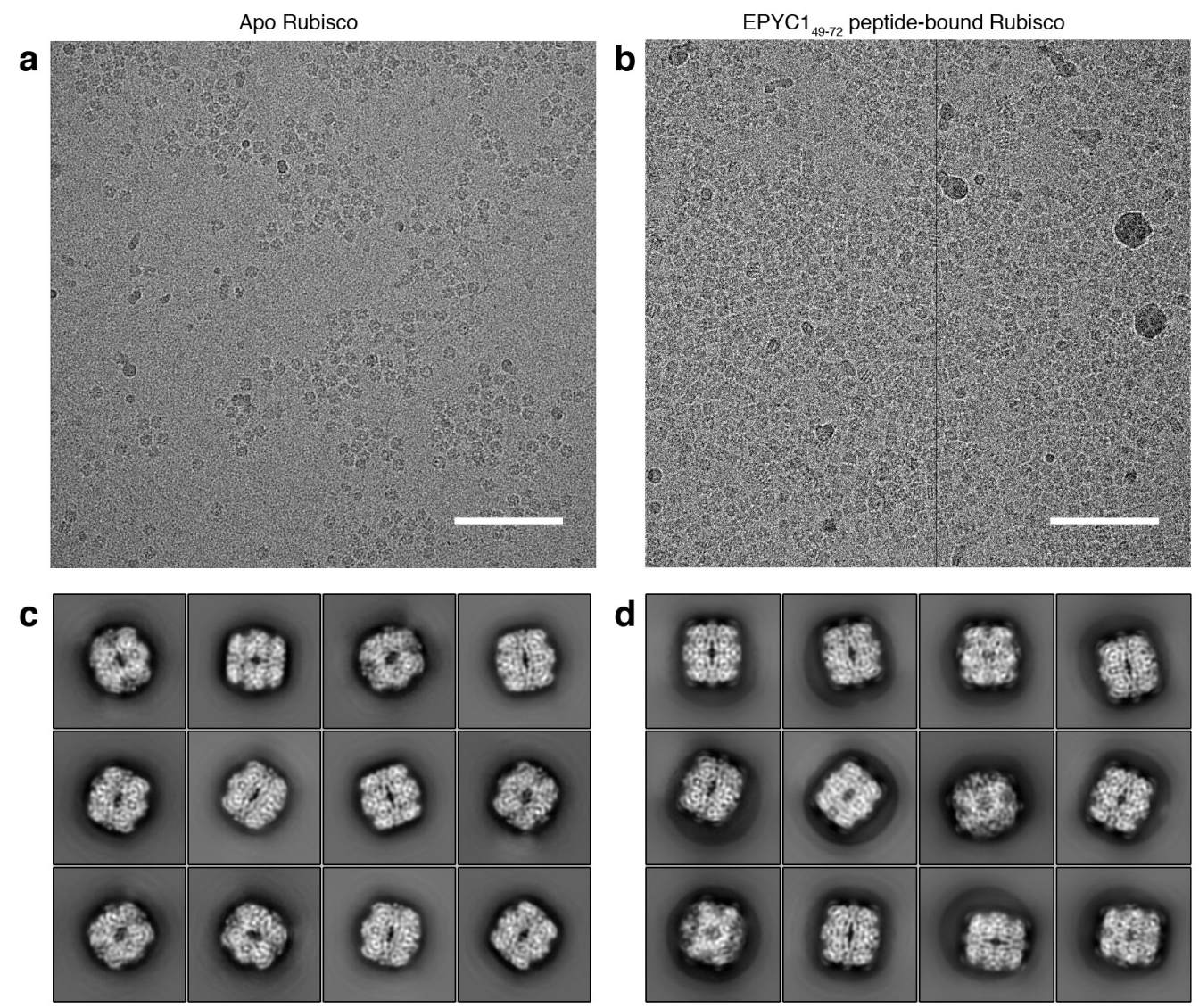

e

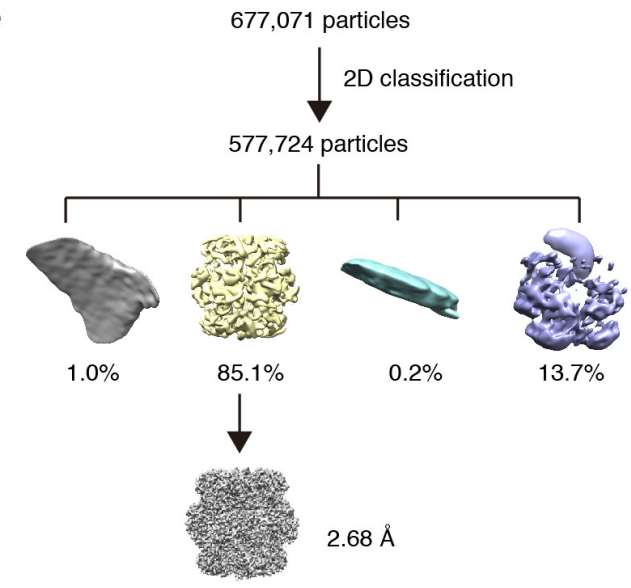

491,395 particles

g

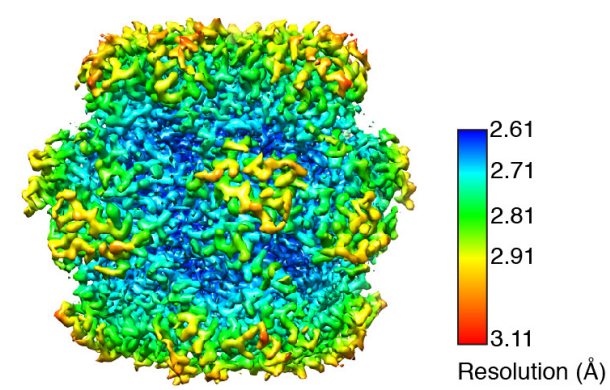

h

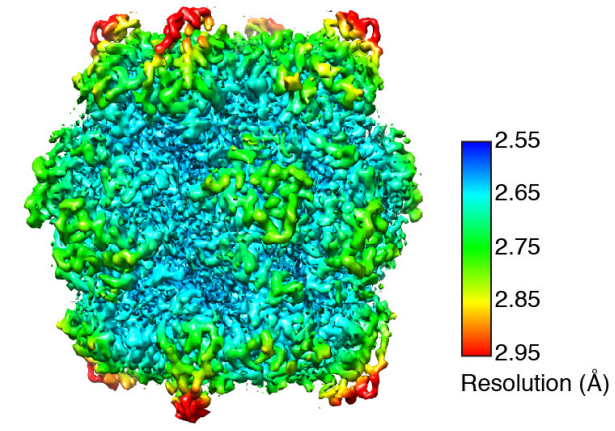

f

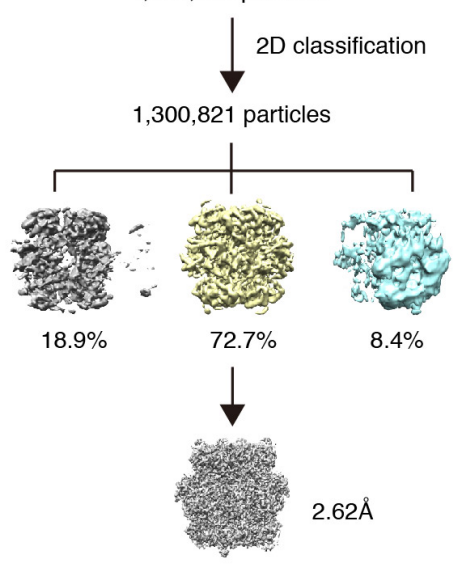

945,755 particles 


\section{Extended Data Fig. 2 | Cryo-EM data collection and image processing procedure. a,}

812 Representative micrograph of the apo Rubisco sample. Scale bar $=100 \mathrm{~nm}$. b, Representative

813 micrograph of Rubisco-EPYC1 ${ }_{49-72}$ complexes. Scale bar $=100 \mathrm{~nm}$. c, Representative $2 \mathrm{D}$ class

814 averages of the apo Rubisco sample. d, Representative 2D class averages of Rubisco-EPYC1 ${ }_{49-72}$

815 complexes. e, Overview of the workflow for single particle data processing for the apo Rubisco

816 sample. f, Overview of the workflow for single particle data processing for the Rubisco-EPYC1 ${ }_{49-}$

81772 sample. g, Local resolution estimation diagram of the final refined apo Rubisco map. h, Local

818 resolution estimation diagram of the final refined Rubisco-EPYC1 $49-72$ complexes map. 
a

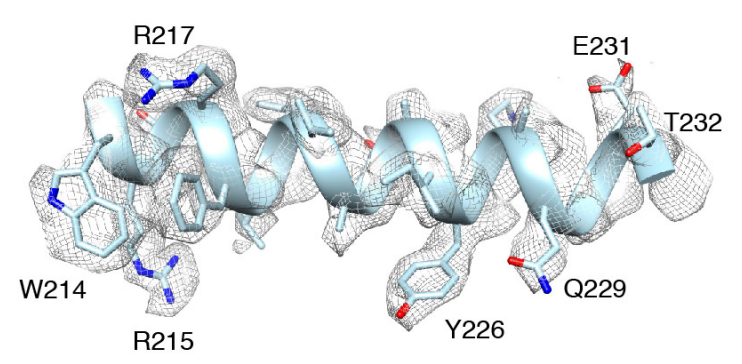

C

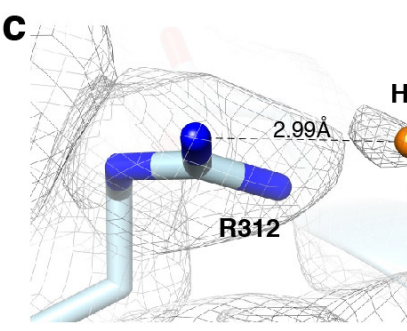

b

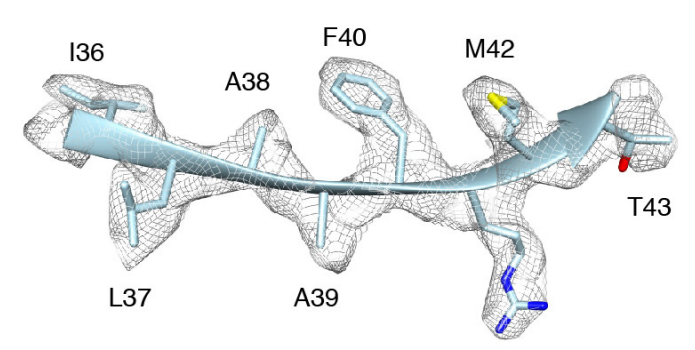

R41

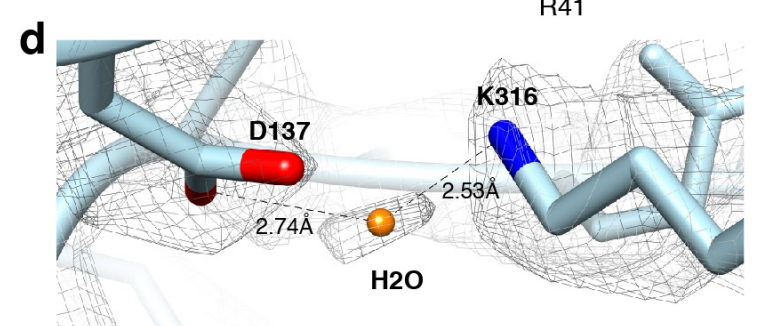

822 complexes in this study. a-b, Representative cryo-EM density quality showing an $\alpha$-helix of

823 residues 214-232 in chain A (a) (one of the Rubisco large subunits) and a $\beta$-sheet of residues 36-

82443 in chain A (b). The densities are shown as meshwork in gray. The backbones of the structural

825 model are in ribbon representation, and side chains are shown in stick representation. c-d,

826 Representative cryo-EM density quality showing water molecules as orange spheres. One water

827 molecule between R312 and E136 on chain A is shown in panel c, and another water molecule 828 between D137 and K316 on chain A is shown in panel d. 
a

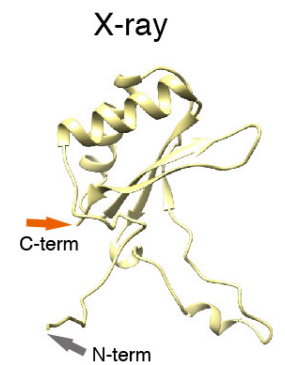

b

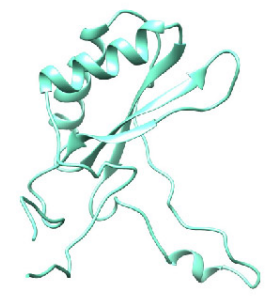

C

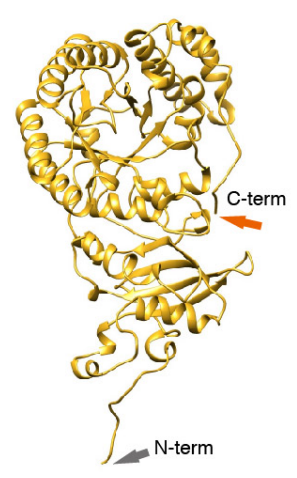

d

EM (apo)

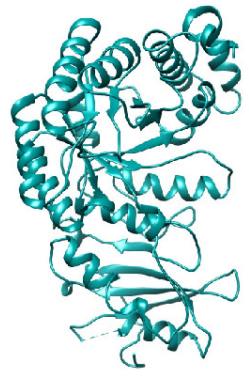

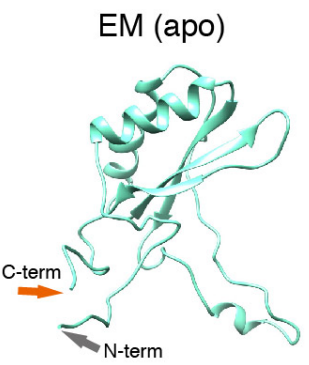

EM (peptide-bound)

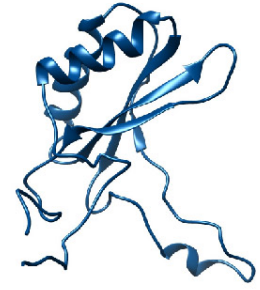

EM (apo)

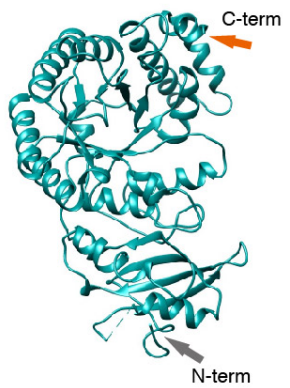

EM (peptide-bound)

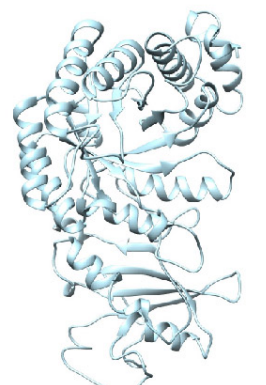

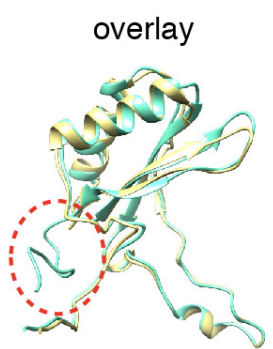

overlay

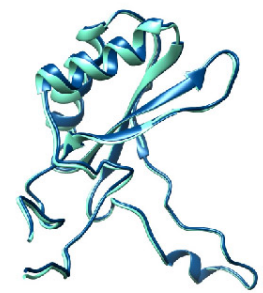

overlay

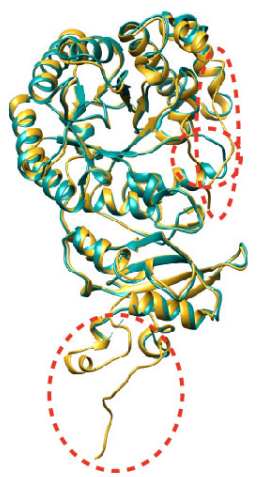

overlay

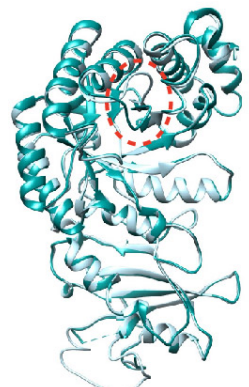

RMSD (X-ray)
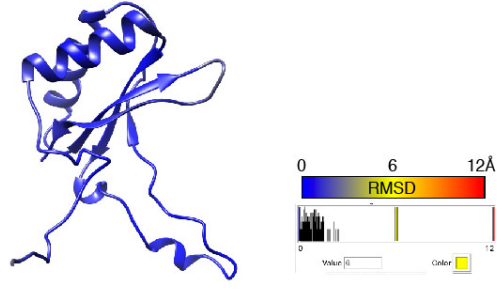

RMSD (EM-apo)
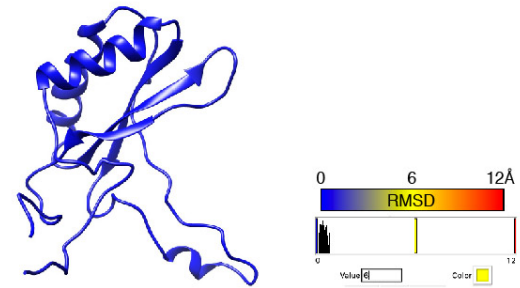

RMSD (X-ray)
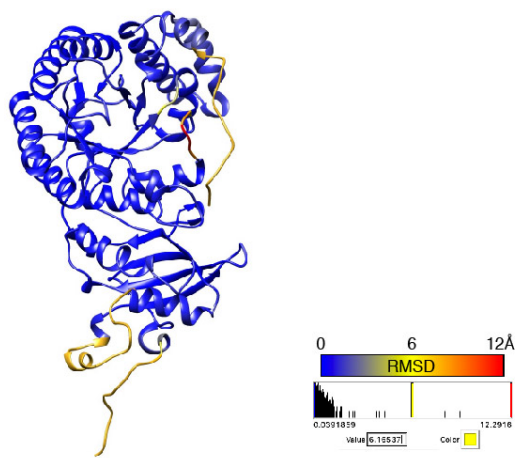

RMSD (EM-apo)

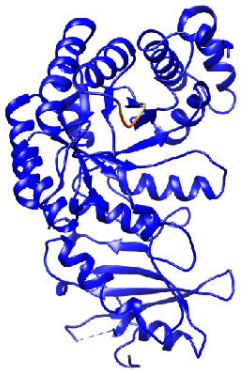

Extended Data Fig. 4 | Comparison of our EM structure and the published X-ray crystallography structure (1gk8) of Rubisco purified from Chlamydomonas reinhardtii ${ }^{13}$, and

834 peptide. a, Comparison of the structure of the small subunit of apo Rubisco obtained here by EM with $1 \mathrm{gk} 8$. The EM structure has additional C-terminus density past residue 126, circled by a red 
836 dashed line. b, Comparison of our two EM structures of the small subunit: from apo Rubisco and

837 from EPYC1 peptide-bound Rubisco. c, Comparison of the structure of the large subunit of apo

838 Rubisco obtained here by EM with $1 \mathrm{gk}$. The three major differences found between the X-ray

839 structure and the EM structure of the large subunit are circled with red dashed lines. d, Comparison

840 of our two EM structures of the large subunit: from apo Rubisco and from EPYC1 peptide-bound

841 Rubisco. The major difference found between the peptide-bound structure and the apo EM

842 structure was the loop between K175 and L180 of the large subunit, which is shown circled by a

843 red dashed line. 

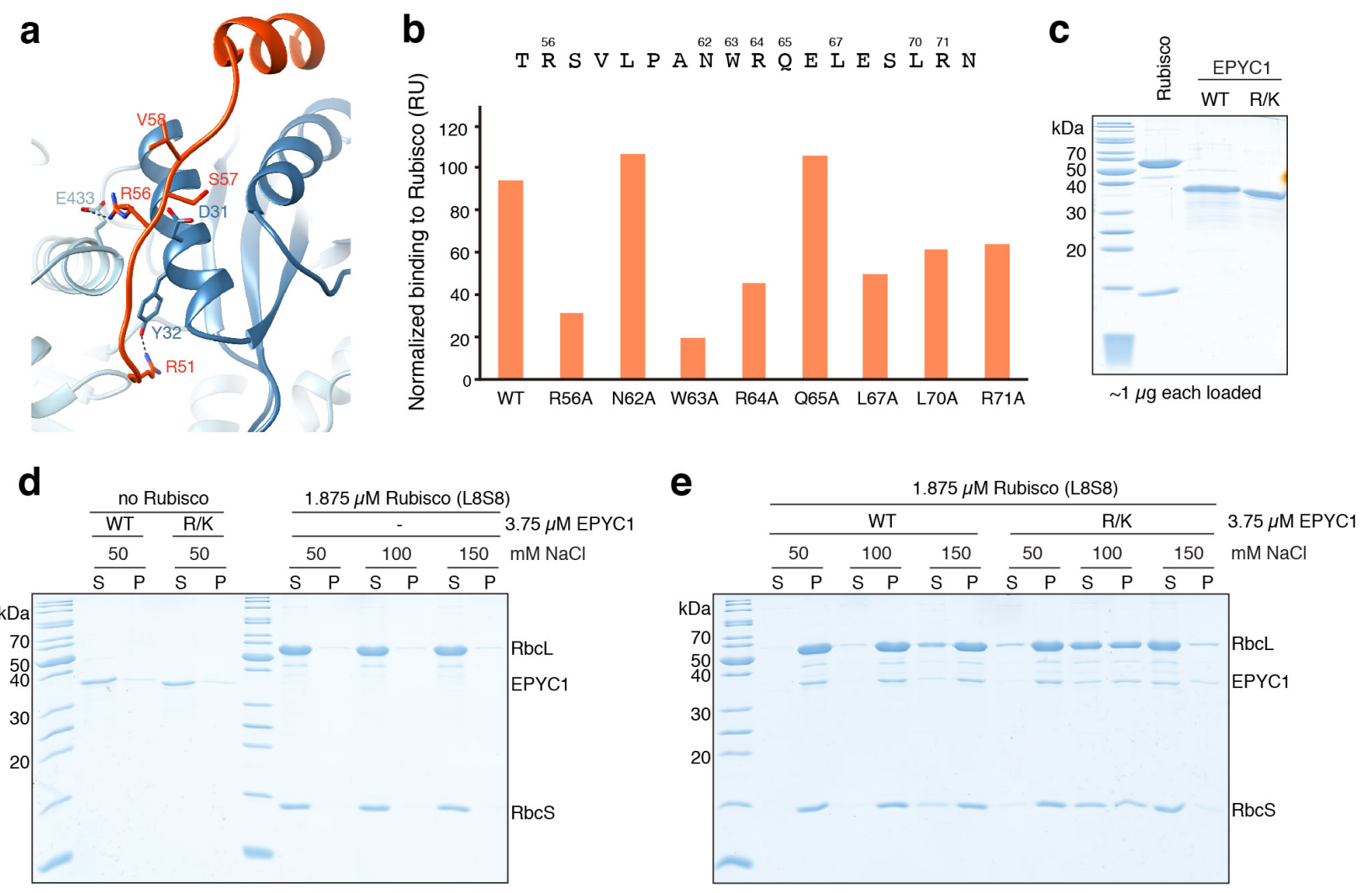

Extended Data Fig. 5 | Interface residues on EPYC1 identified by Cryo-EM are important residues in addition to the ones shown in Fig. 4 may also contribute to the interaction between EPYC1 and Rubisco. R56 of the EPYC1 peptide may interact with both D31 of the Rubisco small subunit and E433 of the Rubisco large subunit. R51 of the EPYC1 peptide may form a salt bridge with Y32 of the Rubisco small subunit. Residues S57 and V58 of the EPYC1 peptide are close to

853 D31 in the structure, which may explain why replacing either of these residues with a negatively

854 charged residue disrupts binding (Fig. 4a). b, The wild-type (WT) EPYC1 peptide or EPYC1

855 peptides with the indicated point mutations were synthesized, and their Rubisco-binding signal 856 was measured by surface plasmon resonance. c, SDS-PAGE analysis of purified proteins used for 857 In vitro phase separation experiments. WT $=$ wild-type $\mathrm{EPYC} 1 ; \mathrm{R} / \mathrm{K}=$ $858 \mathrm{EPYC1} 1^{\mathrm{R} 64 \mathrm{~A} / \mathrm{K} 127 \mathrm{~A} / \mathrm{K} 187 \mathrm{~A} / \mathrm{K} 248 \mathrm{~A} / \mathrm{R} 314}$.d-e, A droplet sedimentation assay was used as a readout of phase 859 separation complementary to the microscopy analyses shown in Fig. 4b. Proteins at indicated concentrations were mixed and incubated for 10 minutes, then condensates were pelleted by 
bioRxiv preprint doi: https://doi.org/10.1101/2020.08.16.252809; this version posted August 16, 2020. The copyright holder for this preprint (which was not certified by peer review) is the author/funder, who has granted bioRxiv a license to display the preprint in perpetuity. It is made available under aCC-BY-NC-ND 4.0 International license.

861 centrifugation. Supernatant $(\mathrm{S})$ and pellet $(\mathrm{P})$ fractions were run on a denaturing gel. The negative

862 controls with no Rubisco or with no EPYC1 are shown in (d), and the wild-type Rubisco with 863 wild-type EPYC1 or mutant EPYC1 are shown in (e). 


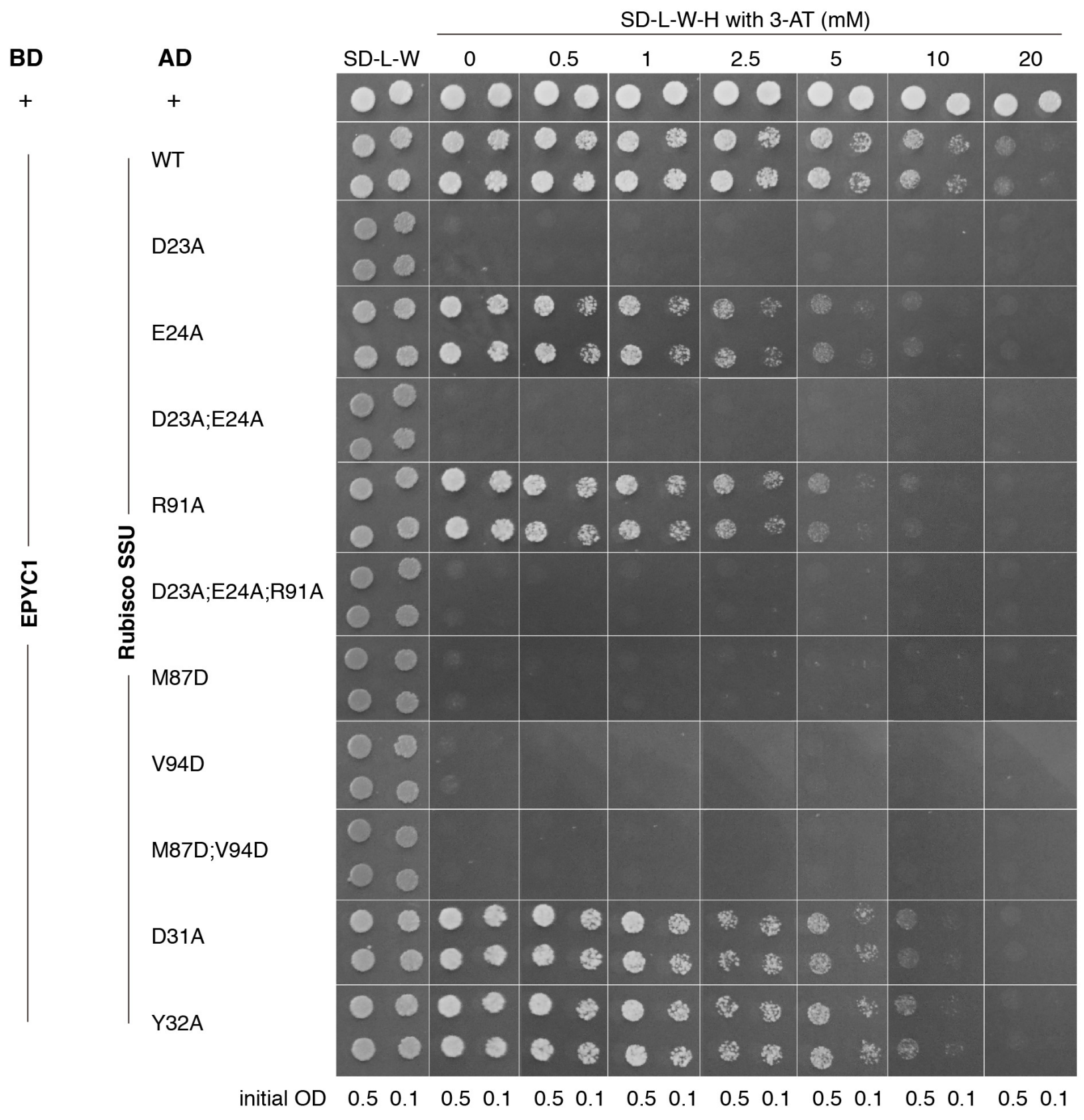

867 Extended Data Fig. 6 | Yeast Two-Hybrid assays of interactions between EPYC1 and wild-

868 type or mutated Rubisco small subunit. Colonies are shown after 3 days' growth on plates. A

869 subset of the data shown in this figure are shown in Fig. 5a. 
bioRxiv preprint doi: https://doi.org/10.1101/2020.08.16.252809; this version posted August 16, 2020. The copyright holder for this preprint (which was not certified by peer review) is the author/funder, who has granted bioRxiv a license to display the preprint in perpetuity. It is made available under aCC-BY-NC-ND 4.0 International license.

a

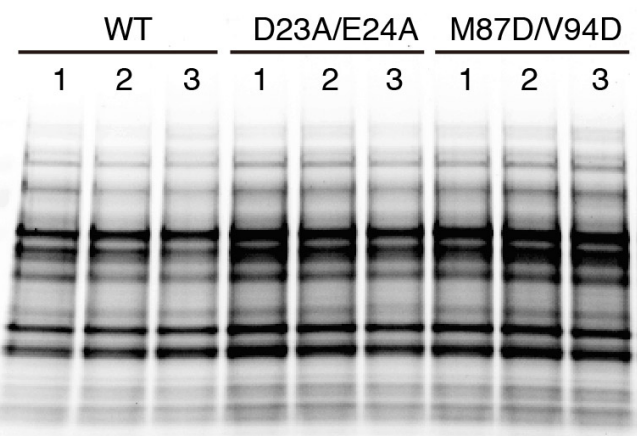

b

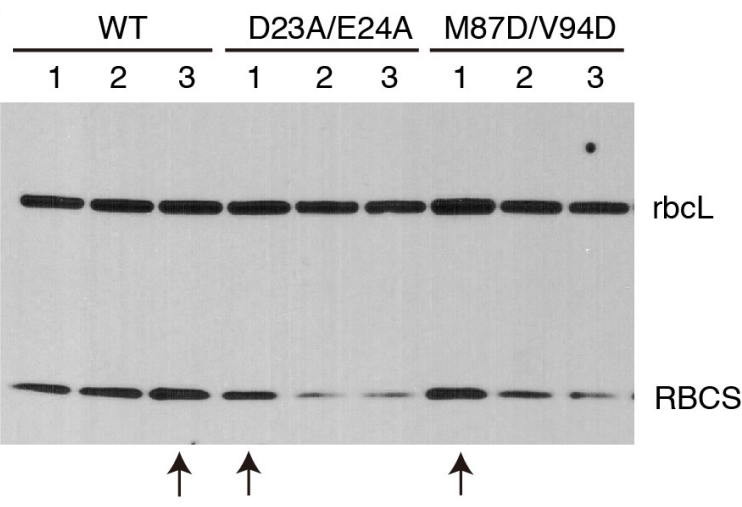

M87D/N94D

C
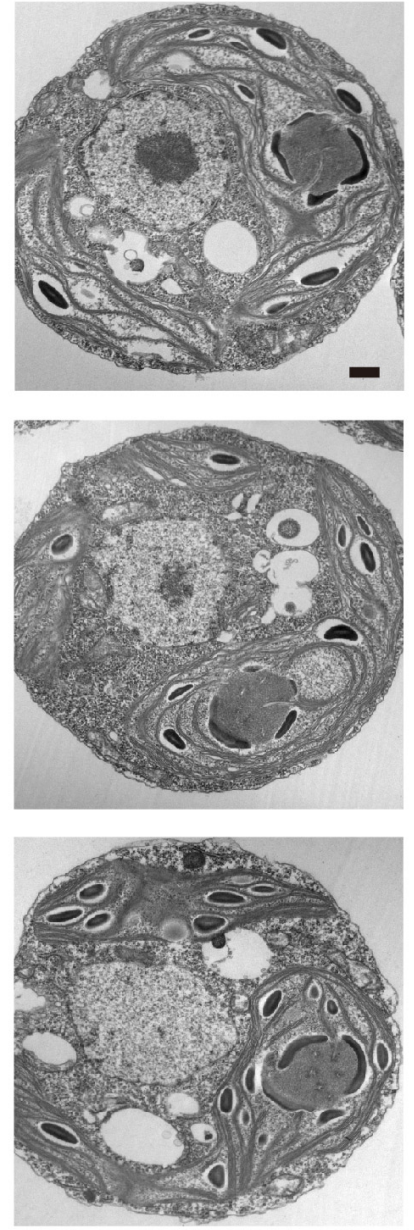

D23A/E24A
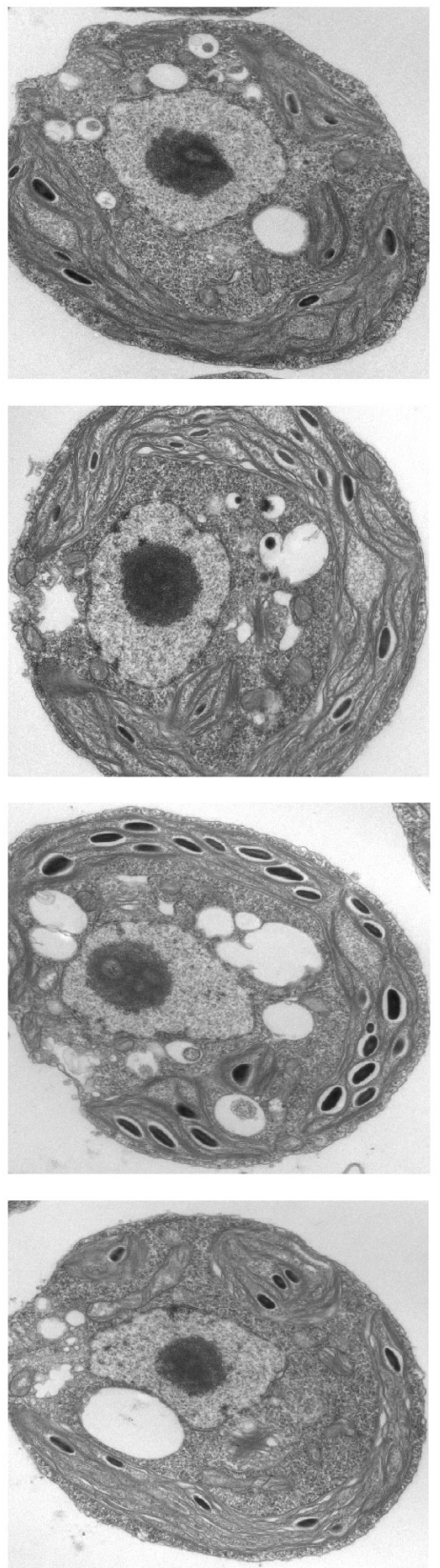
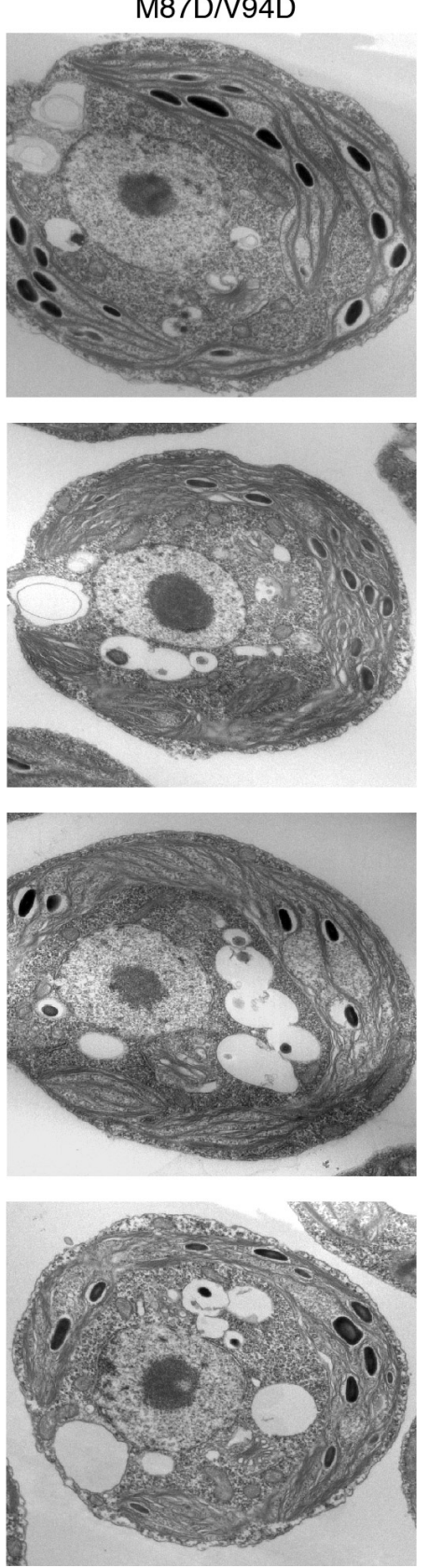


\section{Extended Data Fig. 7 | Selection of the Rubisco small subunit mutant strains for phenotype}

872 analysis. a, The Rubisco small subunit-less mutant T60 ( $\Delta r b c s)$ was transformed with DNA

873 encoding wild-type and mutant Rubisco small subunits (RBCS) to produce candidate

874 transformants with the genotypes $\Delta r b c s ; R B C S^{W T}, \Delta r b c s ; R B C S^{D 23 A / E 24 A}$, and $\triangle r b c s ; R B C S^{M 87 D / V 94 D}$.

875 Total protein extracts for three strains from each transformation were separated on a

876 polyacrylamide gel. b, The gel shown in A was probed by Western Blot using a polyclonal

877 antibody mixture that detects both large and small Rubisco subunits. The candidate transformants

878 with highest RBCS expression level from each genotype are indicated by an arrow below the lanes

879 and were used for the subsequent phenotypic analyses shown in Fig. 5 and panel c. c, Additional

880 representative TEM images of whole cells of the strains expressing wild-type, D23A/E24A, and

881 M87D/V94D Rubisco small subunit. Scale bar $=500 \mathrm{~nm}$. 


\section{Extended Data Table 1 | Cryo-EM data collection and refinement.}

883

\begin{tabular}{|c|c|c|}
\hline & $\begin{array}{l}\text { \#1 Apo Rubisco } \\
\text { (EMDB-xxxx) } \\
\text { (PDB } x x x x)\end{array}$ & $\begin{array}{l}\text { 2\# EPYC1 } 1_{49-72} \text { peptide-bound Rubisco } \\
\text { (EMDB-xxxx) } \\
\text { (PDB xxxx) }\end{array}$ \\
\hline Voltage (kV) & 300 & 300 \\
\hline Magnification & 22,500 & 22,500 \\
\hline Defocus range $(\mu \mathrm{m})$ & -1.5 to -3.0 & -1.5 to -3.0 \\
\hline Pixel size $(\AA)$ & 1.31 & 1.31 \\
\hline Exposure time (s) & 10 & 10 \\
\hline No. movie frames & 50 & 50 \\
\hline Electron dose $\left(e^{-} / \AA^{2}\right)$ & 58 & 58 \\
\hline No. micrographs & 2,500 & 2,500 \\
\hline No. initial particles & 677,071 & $1,809,869$ \\
\hline No. final particle & 491,395 & 945,755 \\
\hline Symmetry & D4 & D4 \\
\hline Resolution (Å) & 2.68 & 2.62 \\
\hline Map sharpening $B$ factor $\left(\AA^{2}\right)$ & -147.233 & -154.161 \\
\hline
\end{tabular}

884 
886 Extended Data Table 2 | The amino acid residues that form the Rubisco-binding regions on

887 EPYC1 homologs, and the EPYC1 binding site on the surface of Rubisco, appear to be

888 conserved across the order Volvocales. Residues with roles in the binding interface are bolded.

889 Residues that are different from the Chlamydomonas reinhardtii sequence are highlighted in grey.

890

\begin{tabular}{cccc}
\hline Species & $\begin{array}{c}\text { First Rubisco-binding region on } \\
\text { EPYC1 homolog }\end{array}$ & Rubisco SSU helix A & Rubisco SSU helix B \\
\hline Chlamydomonas reinhardtii & TRSVLPANWRQELESLRN & DEQIAAQVDYIVA & PMQVLREIVACTKA \\
Tetrabaena socialis & TRSVLPANWRQELESLRG & DEQIAAQVDYIVA & PMQVLREIVSCTRA \\
Gonium pectorale & TRSVLPANWRQELESLRN & DEQIAAQVDYIVA & PMQVLREIVACTKA \\
Volvox carteri & TRSVLPANWRQELESLRN & DEQIAAQVDYIVA & PMQVLREIVACTKA \\
\hline
\end{tabular}

891 